\title{
Graphene-Based Coronal Hybrids for Enhanced Energy Storage
}

\author{
Karthik Kiran Sarigamala $\mathbb{D}^{1,2}$ Shobha Shukla $\mathbb{D}^{2},{ }^{2}$ Alexander Struck $\mathbb{D},^{3}$ \\ and Sumit Saxena $\left.{ }^{2}\right)^{2}$ \\ ${ }^{1}$ Centre for Research in Nanotechnology and Science, Indian Institute of Technology Bombay, 400076, Mumbai, India \\ ${ }^{2}$ Nanostructures Engineering and Modelling Laboratory, Department of Metallurgical Engineering and Materials Science, \\ Indian Institute of Technology Bombay, 400076, Mumbai, India \\ ${ }^{3}$ Faculty of Technology and Bionics, Rhein-Waal University of Applied Sciences, Kleve, Germany 47533
}

Correspondence should be addressed to Sumit Saxena; sumit.saxena@iitb.ac.in

Received 4 September 2020; Accepted 5 January 2021; Published 20 February 2021

Copyright @ 2021 Karthik Kiran Sarigamala et al. Exclusive Licensee Beijing Institute of Technology Press. Distributed under a Creative Commons Attribution License (CC BY 4.0).

\begin{abstract}
Functional materials with designer morphologies are anticipated to be the next generation materials for energy storage applications. In this manuscript, we have developed a holistic approach to enhance the surface area and hence the properties of nanostructures by synthesizing coronal nanohybrids of graphene. These nanohybrids provide distinctive advantages in terms of performance and stability over vertically stacked nanocomposites reported in literature. Various double hydroxide materials self-assembled as coronal lamellae on graphene shells have been synthesized and systematically studied. These coronal nanohybrids result in about a threefold increase in energy storage capacity as compared to their traditionally synthesized nanocomposite counterparts. The 3D graphene-based nanofibrils in the synthesized coronal nanohybrids provide mechanical support and connect the nodes of the double hydroxide lattices to inhibit restacking. Complex morphologies such as coronal nanostructures increase the interaction surface of the nanostructure significantly. Such an approach is also expected to bring a paradigm shift in development of functional materials for various applications such as sensors, energy storage, and catalysis.
\end{abstract}

\section{Introduction}

Engineering morphologies of nanostructures by combining diverse configurations to enhance material properties is an effective approach to synthesize advanced functional materials. The design of novel and sophisticated architectures, however, requires various innovative approaches. Such designer morphologies provide distinctive advantages in terms of performance and stability over traditionally synthesized nanocomposites. Impending innovations in 2D materials are expected to involve expansion of compositional chemistry of interfacial layers [1-3]. Incidentally, incorporation of low-dimensional materials with carbon-based nanostructures is an effective approach for synthesizing materials and offers the combined advantages of both [4-6]. Amongst various low-dimensional materials, 2D materials such as layered double hydroxides (LDHs) have demonstrated great potential to form nanocomposites with many functional nanomaterials [7-12]. These are inorganic clays of layered materials with positively charged brucitelike layers, and they possess several interesting features such as tunability in composition, structure, and morphology. However, there are several challenges associated with the stability and energy storage in nanostructures using LDHs. They aggregate easily $[13,14]$ resulting in a compromised energy storage capacity, lack of conductivity, and instability [15]. This leads to parasitic reactions resulting in constrained electrochemical performance. Thus, a strategic approach needs to be developed to design and synthesize smart material architectures. Recent progress in the synthesis of graphene-based [16, 17] hybrid materials has resulted in a plethora of composites. The major bottleneck is the restacking of the composites with no persistent distinctive nanostructured features [18, 19]. These issues can be mitigated by controlled synthesis of core shell materials to develop distinctive and nontraditional graphene frameworks similar to holey graphene [20] or crumpled graphene nanonetworks [21]. Thus, one can exploit these designer nanohybrids to their full potential [22].

In this perspective, we have developed a holistic approach in designing and synthesizing $3 \mathrm{D}$ coronal architectures of hybrid materials with porous graphene- (PG-) based nanowebs. These obliterate the boundaries between inorganic 
LDH materials that exhibit bulk redox reactions [23-25] and carbon materials that accumulate charge owing to the surface-limited processes [26-28]. These architectures are synthesized with controlled geometry by encapsulating coronal hybrids of ultrathin LDH nanosheets of high redox activity and self-assembled radially overfunctionalized graphene shells, with complementary functionalities. The structural features of coronal hybrids with graphene-based nanostructures introduce reversible wettability and modulation of the chemical potentials with improved rate capability. Additionally, the strain produced in the coronal hybrids due to induced cycling procedures can be relaxed due to the corrugations present on the graphene-based nanonetworks. High-resolution STEM and TEM micrographs show evidence of coronal structural features. Comparative electrochemical studies suggest that synthesized coronal hybrids demonstrate good electrochemical stability and rate capability, better electrochemical performance, and a threefold increase in charge storage as compared to their traditionally synthesized counterparts. Xray diffraction (XRD), X-ray photoelectron spectroscopy (XPS) analysis, and elementally mapped micrographs suggest fine control over the hierarchical structure of the LDH materials. These structures are highly desirable owing to their ability to control the ionic interactions in an electrode-electrolyte interface. Such interactions have been tailored through porous 3D graphene-like nanowebs (3D-PG) which not only prevents restacking but also introduces redox-active organic groups that can electronically communicate with the LDHs. Thus, these nanoarchitectures are expected to create a paradigm shift in the development of materials not only for energy storage applications but also for other applications where surface interactions are extremely crucial, such as catalysis [29, 30], sensors [31, 32], drug delivery [33, 34], and flame retardants $[35,36]$.

\section{Experimental Section}

2.1. Synthesis of 3D-Coronal Hybrids of Co-Mn LDH@3DPG, Ni-Mn LDH@3D-PG, and Ni-Co LDH@3D-PG. Graphene oxide $(\mathrm{GO})$ shells are synthesized using a templateassisted synthesis route, prior to the self-assembly process of coronal lamellae for coronal hybrids. GO solution (detailed procedure for GO synthesis is provided in supplementary information as MT-1 (available here)) is taken and refluxed in a beaker. A mixture of porous silica spheres $(0.8 \mathrm{~g})$ (synthesis of porous $\mathrm{SiO}_{2}$ spheres is detailed in supplementary information as MT-2 (available here)) and DMF solution $(50 \mathrm{~mL})$ is sonicated in a round bottom flask for about 1 hour using an ultraprobe sonicator. Subsequently, (3-aminopropyl)triethoxysilane is added to this flask. The flask is placed in an oil bath, and the temperature of the solution is raised to $110-120^{\circ} \mathrm{C}$. GO solution $\left(1 \mathrm{mg} \mathrm{mL}^{-1}\right)$ along with dicyclohexylcarbodiimide $(2.5 \mathrm{~g})$ is added to this silica suspension. The reaction mixture is then continuously stirred for $24 \mathrm{hrs}$ to obtain core shells of GO coated over silica nanospheres.

To obtain the coronal nanohybrids of LDHs, the GOcoated silica core shell templates were dispersed in $50 \mathrm{~mL}$ alcoholic emulsion and sonicated for about 30 minutes. Hex- amine $(0.56 \mathrm{~g})$ is added to the alcoholic emulsion and sonicated for another 30 minutes. The metallic nitrate precursors (nickel nitrate hexahydrate, cobalt nitrate hexahydrate, or manganese nitrate tetrahydrate) are added for synthesis of their respective LDHs. These are taken with a trivalent metal ratio maintained closely around 0.2-0.3. Later, the obtained solution is sealed in an autoclave reactor vessel with reaction temperature maintained at $90^{\circ} \mathrm{C}$ for 12 hours. The final product is obtained after washing the samples with water and ethanol several times using centrifugation. In order to perform a comparative study, the pristine LDH samples were also prepared under the same conditions of temperature and concentration.

2.2. Characterizations. The surface morphology of the synthesized nanostructures was imaged using a field-emission gun scanning electron microscope (FEG-SEM, JEOL JSM-7600F FEG-SEM) and a high-resolution transmission electron microscope (HR-TEM) equipped with an energy dispersive X-ray spectroscopy (EDS) (Thermo Fisher Scientific, Themis 300 G3) detector. The crystal structure of the samples was investigated using the powdered X-ray diffraction (XRD) technique in the $2 \theta$ range of 5- $80^{\circ}$ at a scan speed of $4 \mathrm{~min}^{-1}$ (Panalytical X'Pert Pro with $\mathrm{Cu} \mathrm{K} \alpha$ irradiation at a wavelength of $0.1542 \mathrm{~nm}$ ). The surface chemical composition and valence states were examined using X-ray photoelectron spectroscopy (XPS) (Axis Supra) with an $\mathrm{Al} \mathrm{K \alpha}$ radiation. To identify the functional groups, FTIR spectroscopy was performed using the 3000 Hyperion Microscope with the Vertex 80 FTIR System (Bruker). The vibrational modes of the samples were investigated using Raman spectroscopy (HR800 UV confocal micro-Raman spectrometer). The $\mathrm{N}_{2}$ adsorption-desorption isotherms and pore size distribution of the samples were measured using a Brunauer-Emmett-Teller (BET) surface analyser (Quantachrome, Autosorb).

The electrochemical studies were performed using a BioLogic SP-300 Potentiostat-Galvanostat. The electrochemical evaluations were carried out using cyclic voltammetry, galvanostatic charge-discharge studies, and impedance spectroscopy using a three-electrode configuration in $3 \mathrm{M}$ aqueous $\mathrm{KOH}$ solution as an electrolyte. A platinum mesh with a crosssectional area of $1 * 1 \mathrm{~cm}^{2}$ and a thickness of $0.1 \mathrm{~mm}$ was used as the counter electrode during the measurements. $\mathrm{Ag} / \mathrm{AgCl}$ was used as a reference electrode for the measurements. The working electrodes were fabricated using the active materials, conductive carbon black, and polyvinylidene fluoride (binding agent) in a weight percentage of $75: 20: 5$, respectively. The slurry made using these ingredients was pasted on to a Ni substrate and dried overnight in a vacuum chamber. The specific capacities were calculated from galvanostatic charge discharge profiles using the following equation: $C_{s}=Q / m$. Here, $C_{s}$ is specific capacity $\left(\mathrm{Cg}^{-1}\right),\left(Q=I_{d} t_{d}\right), t_{d}$ is the discharge period $(\mathrm{s}), I_{d}$ is the constant discharge current (A), and $m$ is the active mass loading of each electrode.

\section{Results and Discussion}

3.1. Biomimetic 3D-Spherical Coronal Hybrids and Characterizations. The synthesis of coronal nanohybrids 


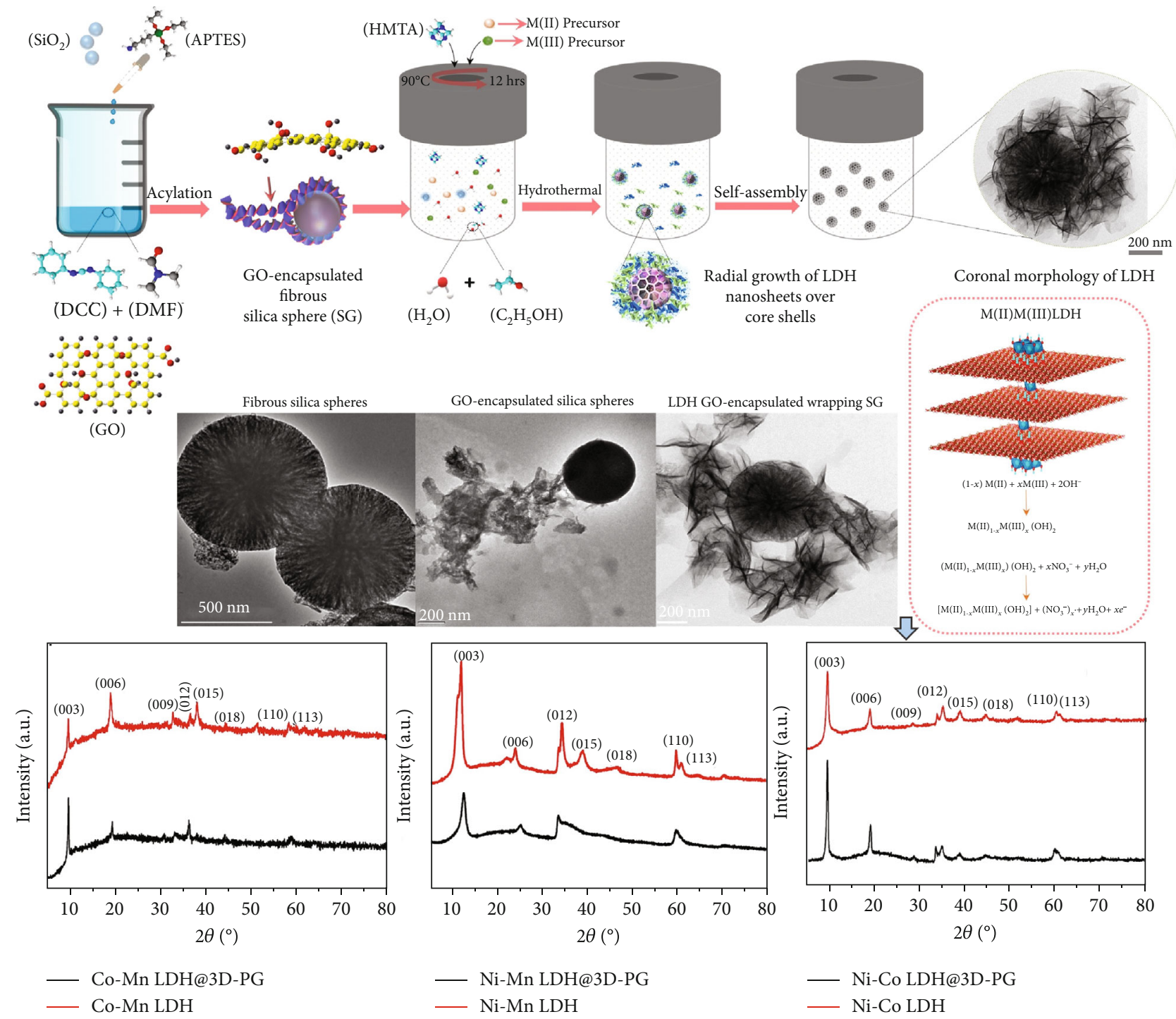

FIGURE 1: Schematic showing synthesis of 3D-networked coronal hybrid nanostructures of LDHs on PG shells using hydrothermal reaction. Synthesis involves growth of LDH lamellae in the form of a corona over the PG shell using surface functionalization forming reduced graphene oxide. Synthesis involves an acylation and encapsulation mechanism via the electrostatic assembly over the fibrous silica particles in the initial stage of the assembly. The final growth stage involves ultrathin LDH nanosheets radially grafted over the PG skeletal structures. High-resolution TEM images are used to understand the synthesis process. The LDH formation mechanism from bivalent and trivalent metallic cations and the XRD patterns of pristine and coronal hybrids of LDHs are presented.

wrapped in intricate nanowebs requires a soft templatebased approach. A schematic showing the detailed synthesis protocol of these nanostructures is shown in Figure 1. In order to achieve this, silica nanoparticles were injected into a solvent medium under agitation with oxidized graphene. This resulted in interfacial growth of GO shells on the silica spheres due to the acylation mechanism and formed uniform dispersion. These were then separated by centrifugation. Subsequently, LDH nanosheets self-assembled during hydrothermal reaction over the $\mathrm{SiO}_{2} / \mathrm{GO}$ core shell structures, and overtime, coronal hybrid assemblies along with 3D fibrillar graphene-like nanowebs were produced. The $\mathrm{SiO}_{2}$ etches out self-sacrificially resulting in the formation of reduced graphene oxide (rGO) shells. This method has been used to demonstrate the synthesis of three representa- tive configurations of transition-metal coronal hybrid structures, i.e., Co-Mn LDH, Ni-Mn LDH, and Ni-Co LDH selfassembled over GO shells embedded in a PG network. A carefully designed porous silica core assists in the structure building and are eventually etched off.

The crystallographic structure of the synthesized hybrid coronal nanostructures was obtained by analysing the XRD pattern. The crystallographic signatures of coronal hybrids are compared with corresponding pristine $\mathrm{LDH}$ nanosheets. The diffraction patterns obtained for the coronal hybrid samples (represented as Co-Mn LDH@3D-PG, Ni-Mn LDH@3D-PG, and Ni-Co LDH@3D-PG) showed features similar to that of a typical hydrotalcite-like structure with the Braggs reflections assigned to the (003), (006), and $(009) /(012)$ planes. These showed good agreement with 
TABLE 1: Calculated lattice parameters and crystallite sizes for the pristine LDH and hybrid samples.

\begin{tabular}{|c|c|c|c|c|c|c|c|}
\hline \multirow{2}{*}{ Sample } & \multicolumn{6}{|c|}{ Lattice parameters $(\AA ̊)$} & \multirow{2}{*}{ Crystallite size (nm) } \\
\hline & $\mathrm{d}_{(003)}$ & $\mathrm{d}_{(006)}$ & $\mathrm{d}_{(009) /(012)}$ & a & c & Basal spacing & \\
\hline Co-Mn LDH & 9.17 & 4.67 & 2.73 & 3.16 & 26.7 & 8.9 & 46 \\
\hline Co-Mn LDH@3D-PG & 9.12 & 4.58 & 2.69 & 3.12 & 26.35 & 8.8 & 35 \\
\hline Ni-Mn LDH & 7.42 & 3.71 & 2.60 & 3.1 & 22.64 & 7.6 & 11 \\
\hline Ni-Mn LDH@3D-PG & 7.16 & 3.57 & 2.53 & 3.08 & 21.89 & 7.3 & 9 \\
\hline Ni-Co LDH & 9.37 & 4.69 & 3.14 & 3.08 & 28.17 & 9.4 & 22 \\
\hline Ni-Co LDH@3D-PG & 9.20 & 4.65 & 3.10 & 3.06 & 27.8 & 9.3 & 15 \\
\hline
\end{tabular}

diffraction patterns of the pristine LDHs (represented as CoMn LDH, Ni-Mn LDH, and Ni-Co LDH) as well. The diffraction patterns for the LDHs exhibited a rhombohedral (3R-type polytype) stacking sequence with $R 3 m$ symmetry. However, the diffraction peaks of graphene-like nanostructures are not apparent in the XRD patterns of the hybrid material because of highly dense coronal LDH lamellae on the surface of ultrathin rGO shells. The lattice parameters along with the tuned basal spacing for various hybrid and pristine LDH samples obtained from XRD measurements have been tabulated in Table 1 below.

The homogenously layered cationic distribution in the synthesized nanostructures is assisted by electrostatic interaction of the cationic layers $[37,38]$. It is understood to be a major factor that favours the crystallization of LDHs. The lattice parameters depend on various factors such as size and amount of interlayer anions, hydration, and cationcation separation $[39,40]$. Further, the presence of possible heterogeneity in finely dispersed crystalline LDHs with some order-disorder in cationic distributions cannot be ignored $[41,42]$. The interplanar spacing is finely tuned with the different compositions. The type of anions intercalated between the interlayers can be controlled by restricting the type and quantity of reagents. The crystallite size obtained from XRD measurements shows that the hybrids have a smaller crystallite size as compared to that of pristine LDHs.

The small LDH crystallites assemble uniformly over the 3D-PG skeletal structure that enables access to all active intercalation sites, leading to highly specific capacities and fast ion diffusion [43]. However, due to the extreme proximity of the LDH nanosheets, the 3D-PG signal was too weak to be observed in XRD.

The surface morphology of representative spherical coronal nanohybrid assemblies of Ni-Mn, Co-Mn, and Ni-Co LDH shown in Figure 2(a) is imaged in STEM mode using high-resolution TEM. The high-resolution STEM micrographs show 3D radial arrangement of nanostructured LDH lamellae radially assembled over the surface of the PG core. After the self-assembly of LDH lamellae on the surface-functionalized graphene, the core shell architecture exhibits the morphology of a spherical coronal structure with a large number of open porous channels which is evident from the FEG-SEM images shown in Figures 2(b) and 2 (c), respectively. The inner layers through chemical moieties present on the backbone of graphene-like nanowebs with a robust $3 \mathrm{D}$ architecture has micro- and mesoporous features that are capable of guiding the electrons along desired and predetermined paths as understood from Figure 2(d). The micrographs also suggest that the LDHs are radially self-assembled on the $\mathrm{rGO}$ shell. During the process of formation of the 3D-PG nanowebs, the remnant oxygen functionalities on graphene sheets act as pillars to provide a relatively large interlayer spacing, while the $\mathrm{sp}^{2}$ clusters that are connected across the interfacial layers form a porous network. Eventually, these nanowebs enhance the ionic current and electronic current conduction rate. In addition to these images, respective FEG-SEM and HR-STEM micrographs for pristine Co-Mn LDH, Ni-Mn LDH, and Ni-Co LDH and hybrid nanostructures of Co-Mn LDH@3D-PG, Ni-Mn LDH@3D-PG, and Ni-Co LDH@3D-PG are shown as Supplementary Figures S1 and S2. Raman spectroscopy is performed to confirm the minor modifications in the properties of the synthesized nanohybrids. Figure 2(e) shows the representative Raman spectra of the Co-Mn LDH@3D-PG, Ni-Mn LDH@3D-PG, and Ni-Co LDH@3D-PG samples. Graphene has two distinctive vibrational modes corresponding to the "D" band $\left(1350 \mathrm{~cm}^{-1}\right)$ which is related to phonons at the " $K$ " point with $A_{1 g}$ symmetry and are linked with structural defects. The " $\mathrm{G}$ " band $\left(1580 \mathrm{~cm}^{-1}\right)$ can be identified with phonons near the zone centre with $E_{2 g}$ symmetry, and this suggests the presence of $\mathrm{sp}^{2}$ hybridized carbons [44]. Raman spectrum for hybrid samples shows the presence of the Raman signature corresponding to graphenelike core structures with " $G$ " and " $D$ " bands. The ratio of intensity of the " $\mathrm{D}$ " to " $\mathrm{G}$ " bands $\left(I_{\mathrm{D}} / I_{\mathrm{G}}\right)$ in all hybrids of LDH@3D-PGs is observed to increase from 0.9 (for the prepared GO) to 1.24 for Co-Mn LDH@3D-PG, 1.38 for NiMn LDH@3D-PG, and 1.31 for Ni-Co LDH@3D-PG, showing the prominence of the architectural composition in size reduction of $\mathrm{sp}^{2}$ in-plane domains. As a consequence, there is also a disorientation in the crystal structure of 3DPG through crosslinking of LDH lamellae [45]. The shifts observed in the Raman spectra suggests that more localized $\mathrm{sp}^{3}$ defects are introduced into the $\mathrm{sp}^{2}$ carbon structure [46]. Briefly, there is an additional disturbance in the $\mathrm{sp}^{2}$ carbon network with the introduction of LDH lamellae. This is expected to exceptionally improve the electrochemical performance through interactive charge transfer between the LDHs and 3D-PGs. These conclusions are also in good agreement with diffraction fringes for the nanohybrids taken at radially aligned locations as shown in Figures 2(f)-2(h) for the three LDH variants. The interplanar spacing calculated from selected area electron diffraction (SAED) patterns obtained using HR-TEM are also indexed to the 


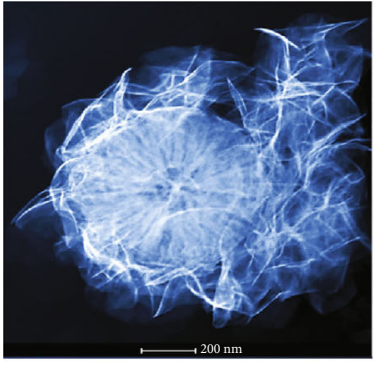

(a)

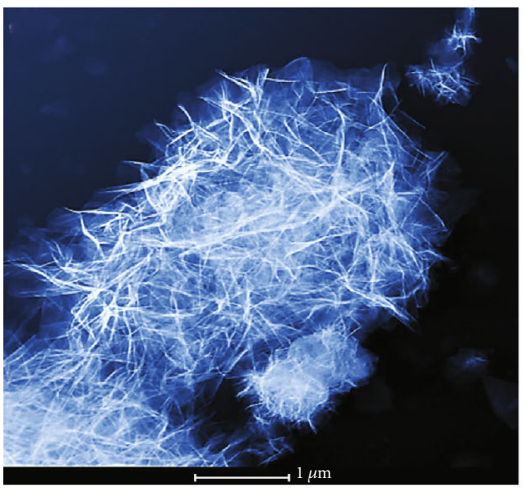

(d)

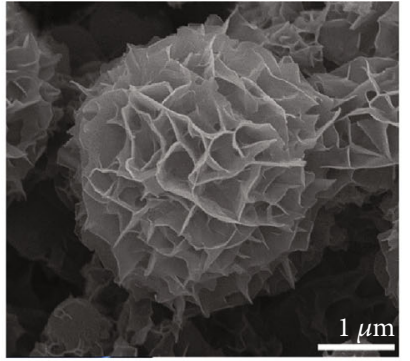

(b)

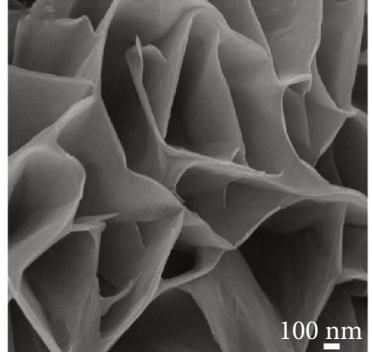

(c)

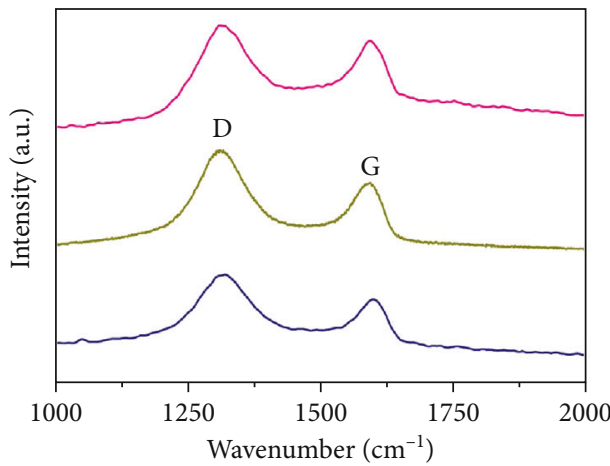

— Co-Mn LDH@3D-PG

— Ni-Mn LDH@3D-PG

_ Ni-Co LDH@3D-PG

(e)

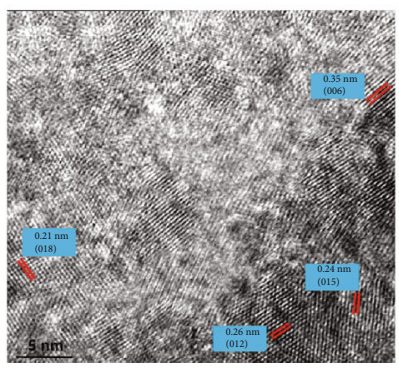

(g)

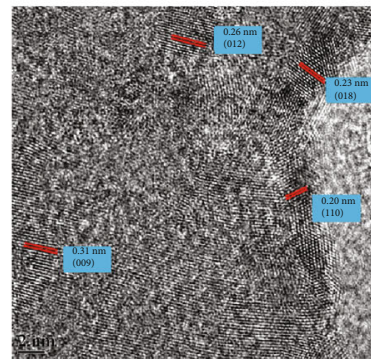

(h)

FIGURE 2: (a) High-resolution STEM image showing assembled spherical coronal LDH nanolamellae over the porous rGO shell. (b) FEGSEM image shows the morphologically stable structure of final spherical coronal nanohybrids. (c) Magnified SEM micrograph showing open porous channels on the surface of the coronal hybrid. (d) High-resolution STEM image shows 3D-PG shells. (e) The Raman spectra for CoMn LDH@3D-PG, Ni-Mn LDH@3D-PG, and Ni-Co LDH@3D-PG are shown. (f-h) High-resolution images showing diffraction fringes matched with their respective lattice spacing for Co-Mn LDH@3D-PG, Ni-Mn LDH@3D-PG, and Ni-Co LDH@3D-PG.

lattice planes of respective LDH hybrids (Supplementary Figure S3). Additionally, the findings from Fourier transform infrared spectroscopy (Supplementary Figure S4) concur with the conclusions drawn from Raman analysis, suggesting the presence of unoxidized graphitic skeletal vibrations and bending mode of carbonyl stretching vibrations of carbon in the hybrid samples.

The homogeneity of elemental distribution in the synthesized samples is investigated using EDS. The elemental compositions for the pristine LDH materials (Co-Mn LDH, Ni-Mn $\mathrm{LDH}$, and Ni-Co LDH) and the hybrid LDH materials (CoMn LDH@3D-PG, Ni-Mn LDH@3D-PG, and Ni-Co LDH@3D-PG) are mapped in Figure 3. The high-resolution STEM mapped regions suggest uniform distribution of the transition elements $(\mathrm{Ni}, \mathrm{Co}$, and $\mathrm{Mn})$ in the $\mathrm{LDH}$ lamellae over the 3D-PG skeletal structures. The pristine LDHs show only the metallic components. The synthesized coronal nanohybrids exhibit the strong signature of the $\mathrm{C}$ and $\mathrm{O}$ elements in the interior portion with branched network-like features which reveal that the core position has no conglomerations as also concluded from the elemental maps. The pristine LDH nanosheets on the other hand aggregate to form large-sized clusters of nanosheets which are crosslinked in a disordered fashion as shown in Supplementary Figure S5. Moreover, the pristine LDH nanosheets look rickety with no open porous structures, which results in poor ionic transfer. These findings are in good agreement with our discussions, and our results provide a significant 


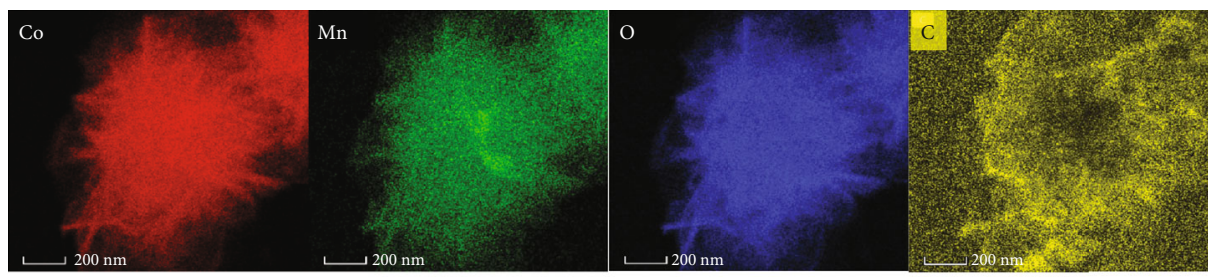

(a)

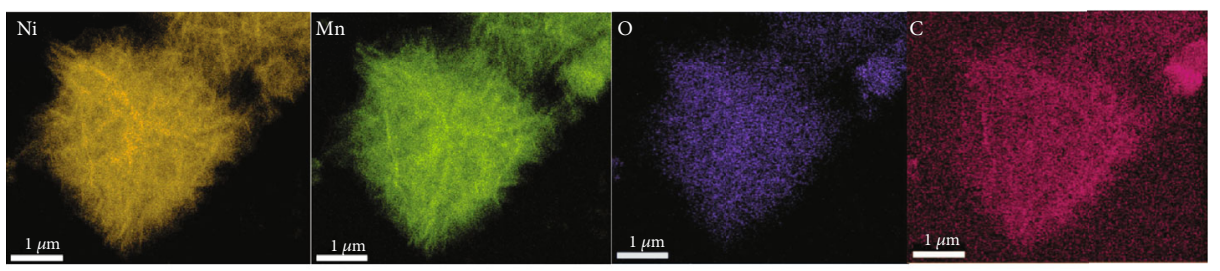

(b)

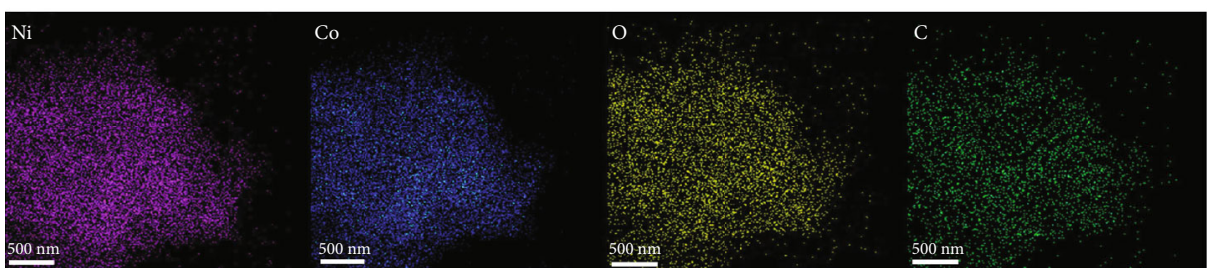

(c)
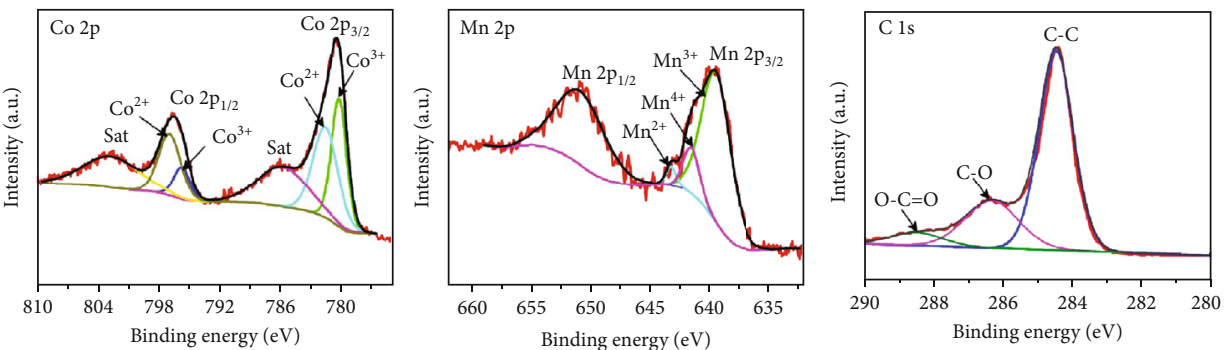

(d)

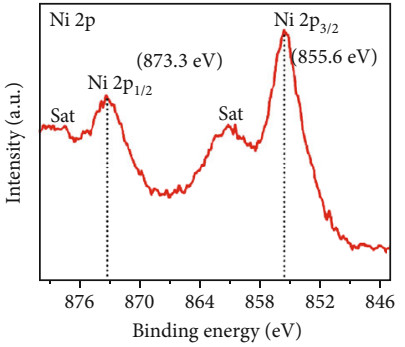

(e)

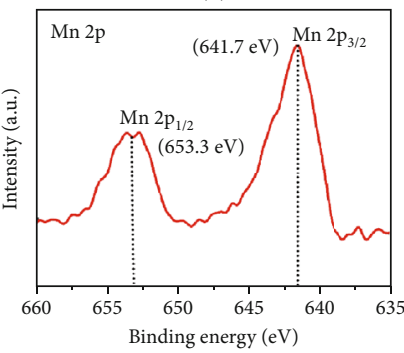

(f)

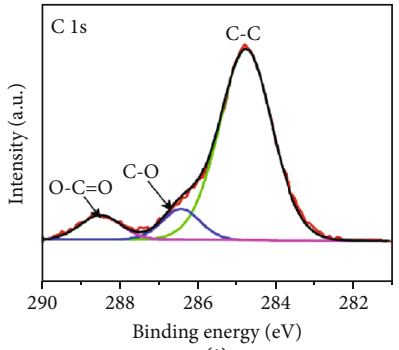

(g)

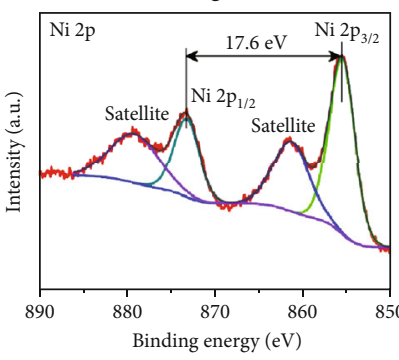

(j)

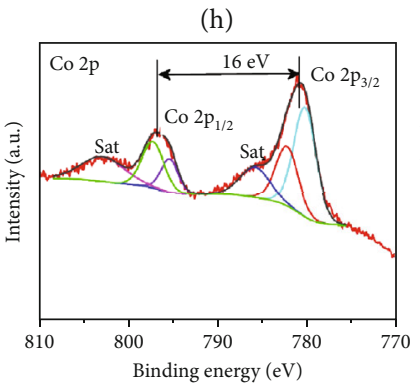

(k)

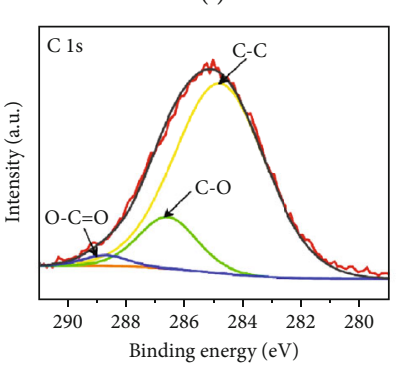

(1)

FIGURE 3: EDS maps for various elements obtained on high-resolution STEM images horizontally aligned from left to right: (a) maps of CoMn LDH@3D-PG; (b) maps of Ni-Mn LDH@3D-PG; (c) maps of Ni-Co LDH@3D-PG. XPS spectra obtained for coronal nanohybrid architectures from left to right: (d-f) XPS spectra for Co-Mn LDH@3D-PG; (g-i) XPS spectra for Ni-Mn LDH@3D-PG; (j-1) XPS spectra for Ni-Co LDH@3D-PG samples. 
improvement over those reported in literature, where the LDHs eventually agglomerate and deposit on the carbon surface (GO or rGO).

High-resolution XPS spectra of all the three coronal hybrids variants were evaluated to investigate the chemical composition as well as the valence states of individual elements. The C1s spectra were deconvoluted to reveal the organic compositional state of graphene oxide core shells. The high-resolution spectra of $\mathrm{Ni} 2 \mathrm{p}$ showed two major peaks besides satellite peaks originating from $\mathrm{Ni}^{2+}$ ion signals at $861.2 \mathrm{eV}$ and $879.1 \mathrm{eV}$. The major peaks associated with both the Ni-based LDH hybrid variants showed peaks at $\sim 855.6 \mathrm{eV}$ and $873.3 \mathrm{eV}$ corresponding to $\mathrm{Ni} 2 \mathrm{p}_{3 / 2}$ and $\mathrm{Ni}$ $2 \mathrm{p}_{1 / 2}$, respectively. The high-resolution Mn XPS spectra in $\mathrm{Ni}-\mathrm{Mn} \mathrm{LDH}$ and Co-Mn LDH hybrids showed two major peaks at $\sim 641.7 \mathrm{eV}$ and $\sim 653.3 \mathrm{eV}$ corresponding to $\mathrm{Mn} 2 \mathrm{p}_{3 /}$ ${ }_{2}$ and $\mathrm{Mn} 2 \mathrm{p}_{1 / 2}$. The valence peaks of $\mathrm{Mn}$ are further deconvoluted, and major contribution from the $\mathrm{Mn}^{3+}$ oxidation state in the samples dominate. It was revealed that additional valence states of $\mathrm{Mn}^{+4}$ and $\mathrm{Mn}^{+2}$ were also included in the spectrum. Moreover, for the Co-based hybrid LDH variants, two pairs of binding energy peaks were observed corresponding to two spin orbit doublets and two satellite shake ups $(\sim 785.9 \mathrm{eV}$ and $\sim 802.9 \mathrm{eV})$. After deconvolution, one pair of binding energies is assigned to $\mathrm{Co}^{3+}$ centred at $\sim 780.2 \mathrm{eV}$ and $\sim 795.4 \mathrm{eV}$ and another pair corresponds to $\mathrm{Co}^{2+}$ centred at $\sim 782.5 \mathrm{eV}$ and $\sim 797.4 \mathrm{eV}$. This confirms multiple oxidation states of the Co species in the hybrid sample. In all the three hybrid samples, C1s originating from graphene oxide core shells, which eventually converts to rGO nanostructures, was clearly validated using the XPS spectra. It has been noticed that graphene oxide is converted to reduced graphene-based nanostructures with strong C-C peaks at $\sim 284.6 \mathrm{eV}$. The deconvoluted spectrum showed peaks with considerably low intensity at $\sim 286.2 \mathrm{eV}(\mathrm{C}-\mathrm{O})$ and $288.5 \mathrm{eV}(\mathrm{O}-\mathrm{C}=\mathrm{O})$ with a smaller proportion confirming effective removal of oxygen functionalities. The reduction rate was understood to be more efficient in Ni-Co LDH@3D-PG compared to the other two hybrid systems. On the other hand, the interaction between morphology, conductivity, and porosity critically defines the physical and electrochemical properties of the resultant controlled nanostructured materials [47]. The isomorphous substitution of bivalent metallic cations by trivalent ions results in a positively charged framework (otherwise called cationic charged framework) [48]. Ionization of oxygenated functional groups (-OH and $-\mathrm{COOH}$ ) in $\mathrm{GO}$ shells resulted in electrostatic interaction due to the difference in electronegativity. Thus, the carbon skeleton strongly binds with LDH lamellae through strong interactions of the cationic metallic framework with oxygen-containing functional groups and the cation- $\pi$ interactions of the same with the $\mathrm{sp}^{2}$ aromatic cluster. Further, the presence of lone pairs of electrons on oxygen atoms in rGO shells allows the rGO shells to accept more protons. Subsequently, LDHs share a proton $(\mathrm{H})$ with the oxygen sites or $\pi$ bands of the rGO core shells, establishing a strong resilience with hydrogen bond [49]. Evidently, there are also weak van der Waals interactions between the LDHs and rGO sheets. Thus, the strong interactions between the LDHs and 3D-
PG protect the 3D architecture. Therefore, even high values of current densities and repeated cycling were unable to break the interactions between the LDHs and the 3D-PG structure with charge storage processes happening due to redox mechanisms in LDHs and charge adsorption/desorption in 3D-PG.

To have a better understanding of the synthesis process of 3D-PG, HR-TEM micrographs were recorded at various stages of synthesis and are shown in Figure 4. Figure 4(a) shows $\mathrm{GO}$ encapsulation over $\mathrm{SiO}_{2}$ templates, while Figure 4(b) shows 3D-PG frameworks obtained from hydrogel-like graphene formed during self-sacrificial removal of silica core. Further, the electron diffraction patterns provided as insets confirmed the transformation of GO nanosheets to rGO-based porous nanostructures. Representative Raman spectrum shown in Figure 4(c) was recorded to support the findings from electron diffraction experiments. Raman data suggests a stacking ratio $\left(I_{\mathrm{D}} / I_{\mathrm{G}}\right)$ of 1.2. FE-SEM micrographs in Figure 4(d) shows 3D open porous structures of graphene-based nanonetworks which show porous channels that help improve the accessible surface area. In conventional graphene-based nanohybrids, the interlayers irrevocably agglomerate due to strong $\pi-\pi$ stacking and van der Waals interactions. This resulted in a reduced surface area. This phenomenon is understood to hinder the ion diffusion properties. Thus, in graphenebased open porous structures, the accessible surface area and the specific capacitance are enhanced. The open channel structures promote the penetration of electrolytes as well as enable an enhanced adsorption of ions. They also help in reducing mechanical stress/strain caused by the electrochemical reactions. Hence, enhancements in electrochemical activity and kinetics are anticipated. The high surface area of graphene along with the presence of substantially large active interfaces are also understood to contribute towards improved performance. Figure 4(e) shows the adsorptiondesorption isotherms derived for both pristine as well as coronal nanohybrid samples for a representative $\mathrm{Ni}$-Co system. The isotherms exhibit type IV characteristics with a hysteresis loop in the $\mathrm{N}_{2}$ desorption branch and appear at a relative pressure of $P / P_{0}>0.4$. This supports the presence of the porous features as concluded from microscopic analysis of our samples.

The surface areas of pristine $\mathrm{Ni}-\mathrm{Co} \mathrm{LDH}$ and $\mathrm{Ni}-\mathrm{Co}$ LDH@3D-PG were calculated to be $20.6 \mathrm{~m}^{2} \mathrm{~g}^{-1}$ and $195.4 \mathrm{~m}^{2} \mathrm{~g}^{-1}$, respectively. Further, the hysteresis loop for the Ni-Co LDH@3D-PG coronal hybrid is of type H3 which is characteristic of a mesoporous structure. In addition, the pore size distribution is provided in Figure 4(f), which shows a large volume of pores in the case of the coronal hybrids. The BET measurements suggest that interfacial contact is established between the $\mathrm{LDH}$ lamellae and the $3 \mathrm{D}$ graphene structures enabling an efficient charge transfer mechanism by reducing the ion diffusion length.

3.2. Charge Storage Mechanism in the Coronal Hybrid Architecture. The 3D-PG nanonetworks ease the electrolytic access and enhance the overall specific charge storage capacity compared to the solid matrix or aggregated sheets of the 


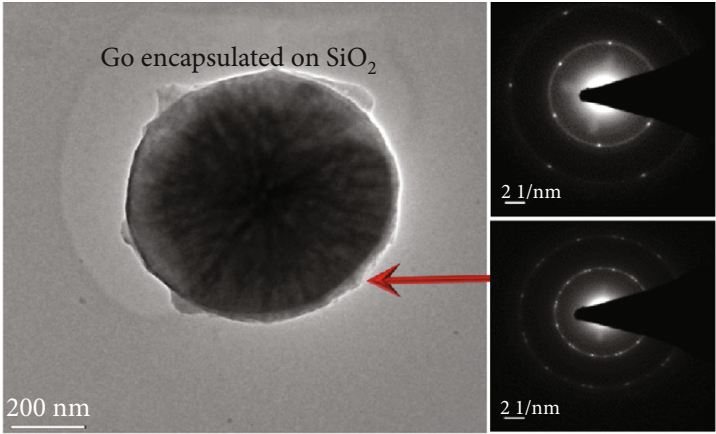

(a)

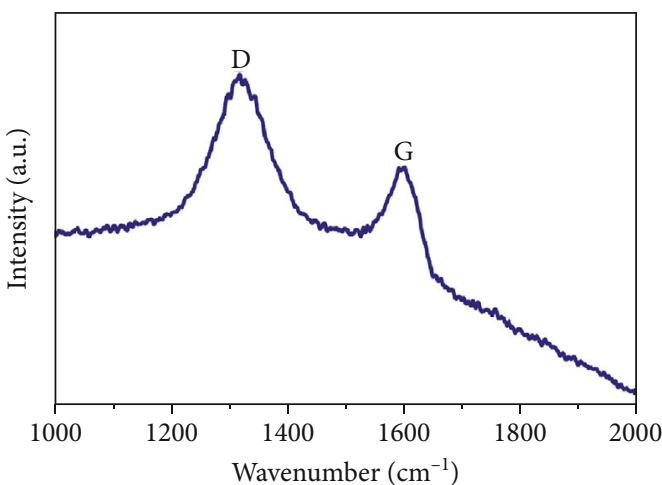

_ Raman spectrum

(c)

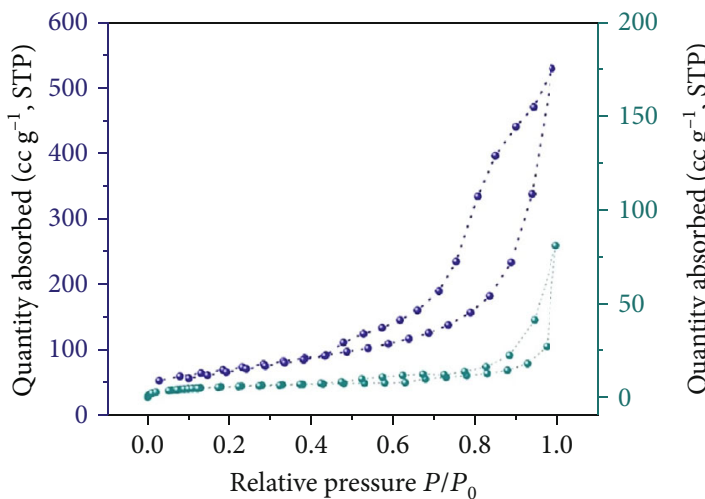

$\multimap$ Ni-Co LDH@3D-PG

$\multimap \mathrm{Ni}-\mathrm{Co} \mathrm{LDH}$

(e)

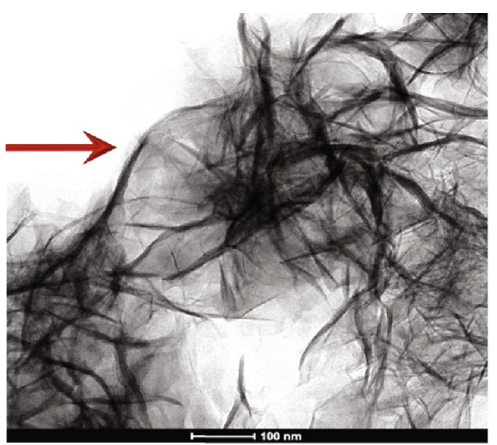

(b)

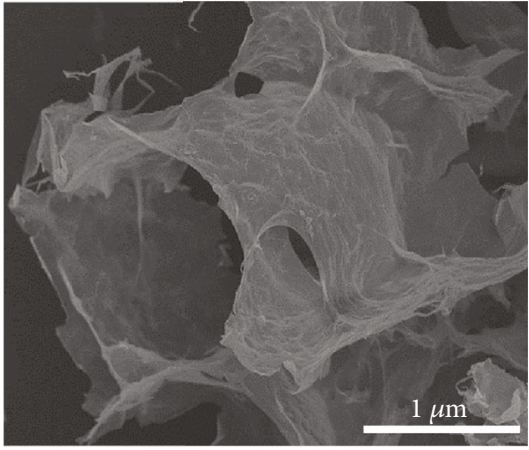

(d)

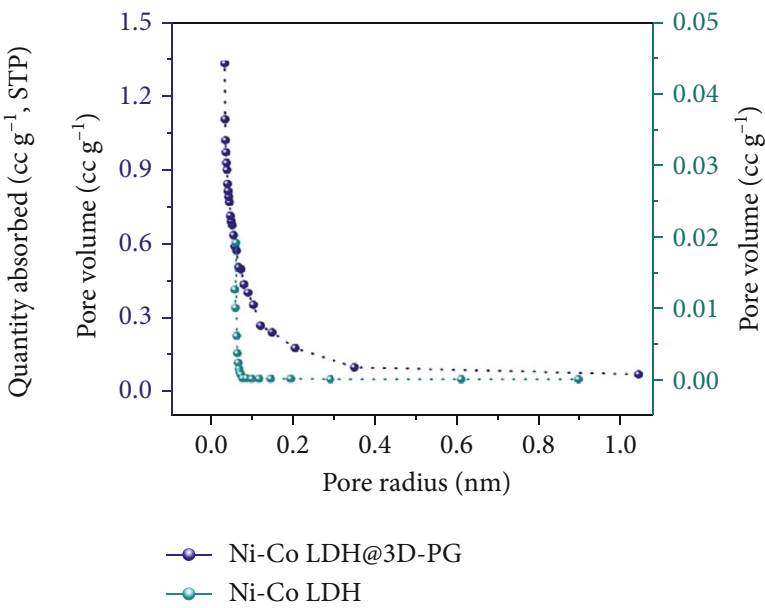

(f)

FIgURE 4: (a) HR-TEM micrograph showing GO-encapsulated $\mathrm{SiO}_{2}$. Bottom right inset shows the SAED pattern obtained from the surface indicated with a red arrow pointing towards the left. (b) HR-TEM image of 3D porous graphene obtained via hydrogel formation during a self-sacrificial process. Top right inset shows the SAED pattern obtained from the thin surface indicated with a red arrow pointing towards the right. (c) Raman spectrum of a 3D porous graphene structure. (d) FE-SEM image of the 3D porous structure of graphene extracted from the $\mathrm{GO} / \mathrm{SiO}_{2}$ core. (e) Comparison of the $\mathrm{N}_{2}$ adsorption/desorption isotherms of a pristine Ni-Co LDH and a coronal nanohybrid of Ni-Co LDH @3D-PG. (f) Comparison of the pore size distribution of a pristine Ni-Co LDH and a coronal nanohybrid of Ni-Co LDH @3D-PG.

active material as shown in Figure 5(a). The biomimetic 3D graphene backbone provides a good surface area with open porous channels. These enhance the redox-active sites and fast transport of ions. This makes the GO-coated silica core shells an ideal template for making hybrid nanostructures. The LDH compositions used in synthesis are typically of the battery-type hybrid that exhibits pseudocapacitive behaviour. The self-assembly of LDH nanosheets on the 3D-PG skeletal structure enables the formation of ordered nanostructures. Transition-metal LDHs not only offer much larger charge storage but most of them also have low electrical conductivity. The interconnected graphene nanowebs 

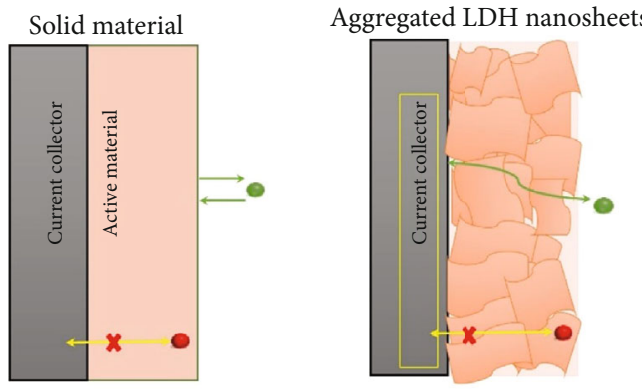

UItrathin LDH woven over 3D-PG nanowebs

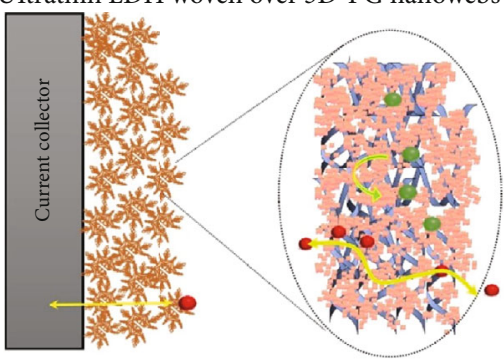

(a)

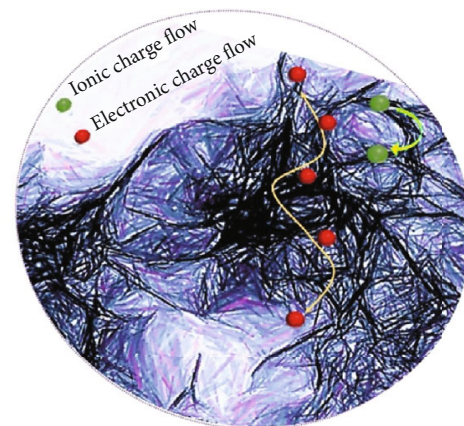

Guided paths for ionic and electronic charge Transport through graphene-based nanowebs

(b)

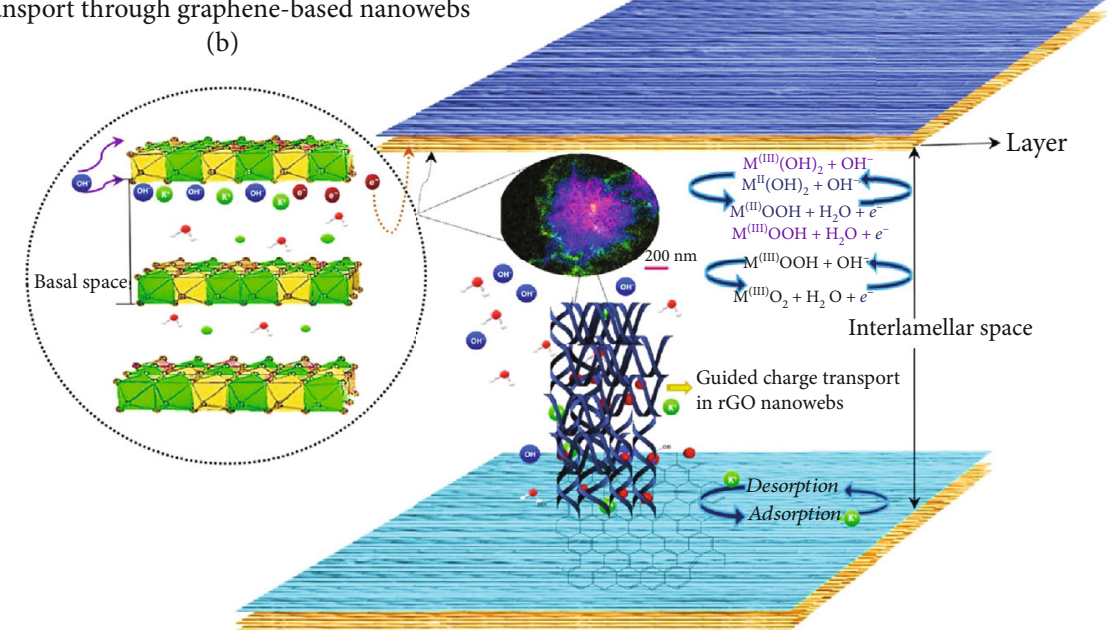

(d)

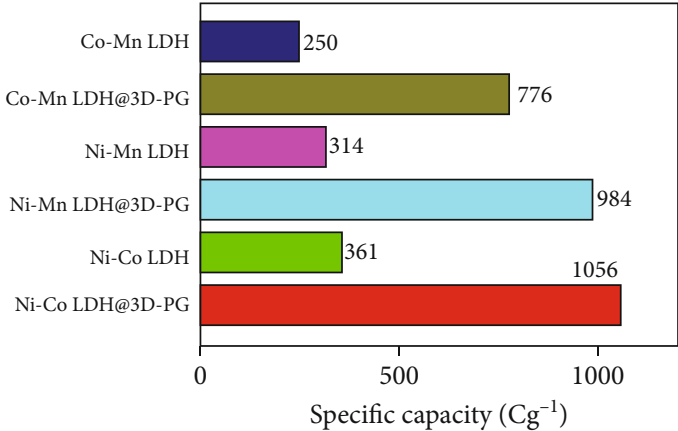

(c)

FIGURE 5: (a) Schematic showing ionic and electron charge transfer in solid active material, aggregated LDH nanosheets, and hybrid LDHs over 3D-PG nanowebs. (b) Graphics showing distribution of nanowebs inside the coronal hybrid and illustrative charge transfer pathways. (c) Comparison of specific capacities of pristine LDHs with their respective coronal nanohybrids. (d) Schematic illustrating various components inside a graphene-based coronal hybrid.

provide high electrical conductivity and chemical stability by synergising the virtues of active materials into a hybrid structure as suggested in Figure 5(b). Moreover, compared to traditional solid and aggregated morphologies, the synthesized coronal nanohybrids aid in shortening the diffusion path of the ions because of their large aspect ratio. They also help in effective utilization of the active materials. Thus, the coronal nanohybrids with carbon skeletal frameworks act as mechanical support.

It assists in the dispersion of the $\mathrm{LDH}$ nanosheets to avoid self-aggregation. This helps in creating extra active sites which help in improving the electrochemical performance. Hence, the specific capacity of the hybrid materials is observed approximately three times higher than the pristine LDH materials as seen in Figure 5(c). In addition to offering high specific capacity, the perforations in the coronal structures as shown in Figure 4(d) and the nanowebshaped supports significantly improve the mechanical strength as compared to solid films of the pristine material. Charges are stored from within the electrode bulk to the surface involving redox processes that occur at orders of magnitude faster and allow greatly improved power densities and enhanced cycle life [50]. The redox reactions involving LDHs with represented bivalent metal ion $\left(\mathrm{M}^{\mathrm{II}}\right)$ and trivalent metallic ion $\left(\mathrm{M}^{\mathrm{III}}\right)$ configurations transform into oxyhydroxides or/and to oxide under a reversible redox mechanism with 
$\mathrm{Ni}, \mathrm{Mn}$, or Co. Additionally, there is an adsorption and desorption phenomenon taking place in the 3D-PG framework shown in Figure 5(d). The high electronic and ionic conductivities combined with intrinsic strength and flexibility of graphene nanowebs allow structural stability for enhanced energy storage.

3.3. Electrochemical Evaluation of the Hybrid Materials. The multilayered architecture of coronal hybrids significantly enhances the specific surface area along with the porosity. This provides abundant active sites for the migration of ionic species and stimulates the interaction between the electrolyte and active material [51]. Consequently, the coronal hybrids demonstrate higher specific capacity and rate performance compared to the pristine materials. Electrochemical evaluation and investigation of pristine $\mathrm{Co}-\mathrm{Mn} \mathrm{LDH}, \mathrm{Ni}-\mathrm{Mn}$ $\mathrm{LDH}, \mathrm{Ni}-\mathrm{Co} \mathrm{LDH}$, and their respective coronal nanohybrids have been conducted in a three-electrode configuration using cyclic voltammetry (CV), galvanostatic chargedischarge (GCD), and electrochemical impedance spectroscopy (EIS) measurements. A $3 \mathrm{M} \mathrm{KOH}$ solution is used as an electrolyte. Comparisons of the representative cyclic voltammograms of synthesized pristine LDHs and their coronal nanohybrids are shown in Figures 6(a)-6(c). The CV curves of pristine $\mathrm{Co}-\mathrm{Mn} \mathrm{LDH}, \mathrm{Ni}-\mathrm{Mn} \mathrm{LDH}, \mathrm{Ni}-\mathrm{Co} \mathrm{LDH}$, and their coronal nanohybrids were obtained at a scan rate of $5 \mathrm{mVs}^{-1}$. These show redox peaks during the anodic and cathodic sweeps suggesting typical pseudocapacitive behaviour. The CV curves of pristine LDHs and their hybrids show similar profiles, suggesting the occurrence of identical electrochemical processes. The CV curves for the coronal nanohybrid exhibit a large area under the curve suggesting enhanced energy storage. The general equations for the redox processes in the $\mathrm{LDH}$ hybrids are shown in Figure 5(d). The Co-Mn LDH and Co-Mn LDH@3D-PG have two redox couples within the potential window of $-0.2 \mathrm{~V}$ to $0.6 \mathrm{~V}$. Two anodic peaks are observed at $\sim 0.1 \mathrm{~V}$ and $\sim 0.46$ along with two cathodic peaks at $\sim 0.02$ and 0.36 . The first redox couple is due to a reversible Faradaic transition from the $\mathrm{Mn}^{2+} / \mathrm{Mn}^{3+}$ couple, and the asymmetric peaks can be associated with a reversible transition from $\mathrm{Mn}^{3+}$ to $\mathrm{Mn}^{4+}$. The second redox couple is associated with quasireversible conversion of $\left(\mathrm{Co}^{2+} / \mathrm{Co}^{3+}\right)$ and $\left(\mathrm{Co}^{3+} /\right.$ $\left.\mathrm{Co}^{4+}\right)$. A pair of redox peaks is also obvious for $\mathrm{Ni}-\mathrm{Mn}$ LDH and Ni-Mn LDH@3D-PG signifying an active redox environment in these battery-type hybrid materials. Similarly, the area enclosed under the CV curve and the peak current for the Ni-Mn LDH coronal nanohybrid is much larger as compared to that of pristine Ni-Mn LDH. It is evident that in Ni-Mn LDH@3D-PG, most of the energy storage and current are achieved in the potential range of 0.2$0.55 \mathrm{~V}$. The net charge stored in the synthesized coronal hybrids also depends on the charge stored not only on the outer surface (i.e., due to LDH lamellae) but also in the inner surface region accessed between the electrolyte and active electrode. The high redox currents with a large enclosed area under the $\mathrm{CV}$ curve are realized from reasonable compositional tuning of the coronal hybrids. For instance, the coronal hybrid of Co-Mn LDH and Ni-Mn LDH delivered a high specific capacity due to an optimum replacement of Co or $\mathrm{Ni}$ by $\mathrm{Mn}$. It is also understood that the structural mitigation by tailoring coronal hybrids using 3D graphene-based nanowebs can help generate a good concentration gradient. However, Co-Mn LDH@3D-PG delivers a low specific capacity value than Ni-Mn LDH@3D-PG which may be due to the highly active $\mathrm{Ni}^{2+}$. During the in situ growth process, GO shells oxidize $\mathrm{Co}^{2+}$ and $\mathrm{Mn}^{2+}$ to trivalent metallic ions, but $\mathrm{Ni}^{2+}$ still remains more stable and assists in the enhancement of specific capacity and rate capability. In the case of Ni-Co LDH and Ni-Co LDH@3D-PG, both cyclic voltammograms show a pair of redox peaks. Due to the Faradaic reactions, $\mathrm{Ni}(\mathrm{OH})_{2}$ undergoes transition from $\mathrm{Ni}^{2+}$ to $\mathrm{Ni}^{3+}$. Additionally, two pairs of peaks arise from $\mathrm{Co}(\mathrm{OH})_{2}$ due to redox transition from $\mathrm{Co}^{2+}$ to $\mathrm{Co}^{3+}$ and $\mathrm{Co}^{3+}$ to $\mathrm{Co}^{4+}$. The redox peaks are quasireversible with a good capacitive behaviour. Most of the charge storage is predominant in the potential range of $0.1-0.4 \mathrm{~V}$. In general, the total charge storage enclosed under the $\mathrm{CV}$ curves for the hybrids can be governed by three mechanisms: (a) the pseudocapacitive charge storage mechanism due to the intercalation/deintercalation of electrolytic ions, where both $\mathrm{OH}^{-}$ions and $\mathrm{K}^{+}$ ions intercalate/deintercalate into the interlayer domains of the LDHs which is controlled by the diffusion-limited process [52]; (b) the redox reactions over the cationic surfaces of LDHs by electroactive species; and (c) the double-layer charge storage component, due to charge adsorption/ desorption of ions over the 3D-PG frameworks, which could be assigned as a surface-limited process [53]. The redox peak shifts (anodic peak shift to a higher potential and cathodic peak shift to a lower potential) of LDH@3D-PGs indicate the effect of polarization, which can be attributed due to the phase separation between pristine LDHs and the presence of the 3D graphene framework in LDH@3D-PGs. However, it is anticipated that the presence of the carbon framework decreases the polarization by improving the electronic conductivity. The other reason behind the peak separation is due to the overall concentration gradient of $\mathrm{Ni}$ or Co affected by the presence of GO during the 3D-PG formation [54]. Further, to realize the effect of 3D-PGs and to measure the specific capacities of the pristine LDHs and their hybrids, the samples were characterized using GCD measurements. The specific capacities of all the electrodes were calculated from the charge-discharge curves. A comparative study of the GCD profiles for pristine and coronal hybrids is shown in Figures 6(d)-6(f). The study was performed in $3 \mathrm{M} \mathrm{KOH}$ solution at a current density of $1 \mathrm{Ag}^{-1}$. The observed potential vs. time response for the synthesized LDH configurations suggests that the Faradaic reactions are highly reversible with a charge-discharge coulombic efficiency of more than $~ 99 \%$. A symmetric triangular profile with well-defined plateaus suggests good capacitive behaviour. One portion of the profile shows a linear discharge profile, indicating a surface-limited charge storage process. The broad extended plateau region represents a major portion of charge storage during discharge due to the redox-active species with a low polarization.

The notable deviation in the GCD profiles from a straight line is understood to occur primarily due to the redox reaction mechanisms of nickel or cobalt species. The specific capacities of Co-Mn LDH, Ni-Mn LDH, and Ni-Co LDH calculated 


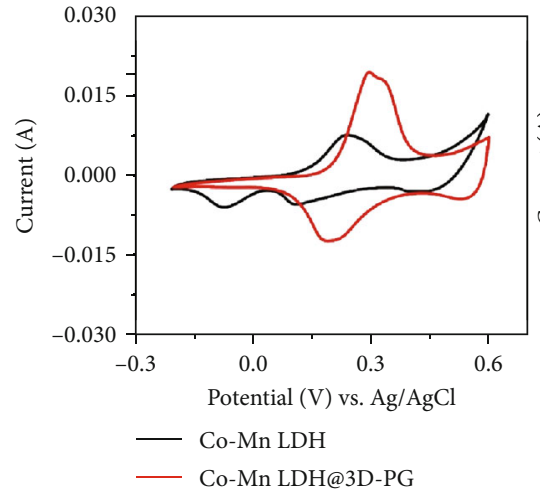

(a)

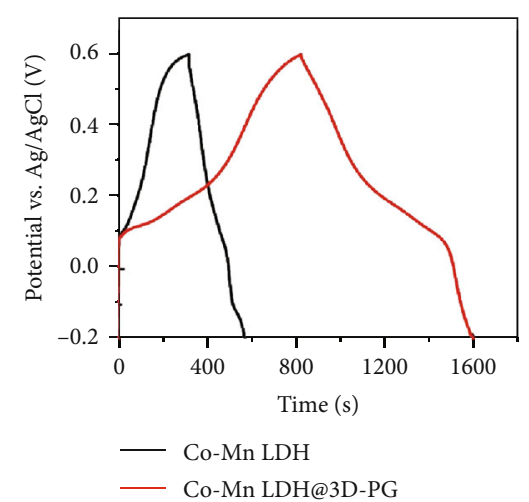

(d)

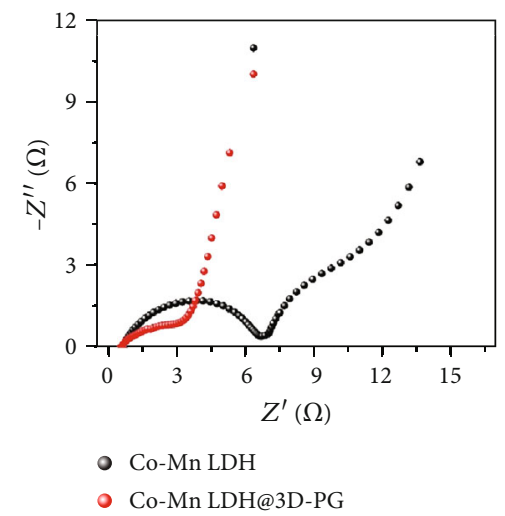

(g)

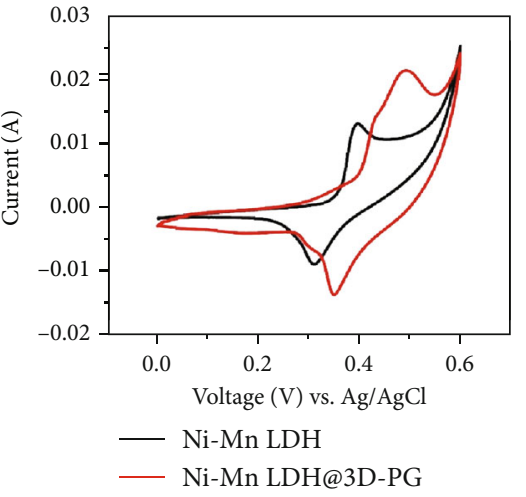

(b)

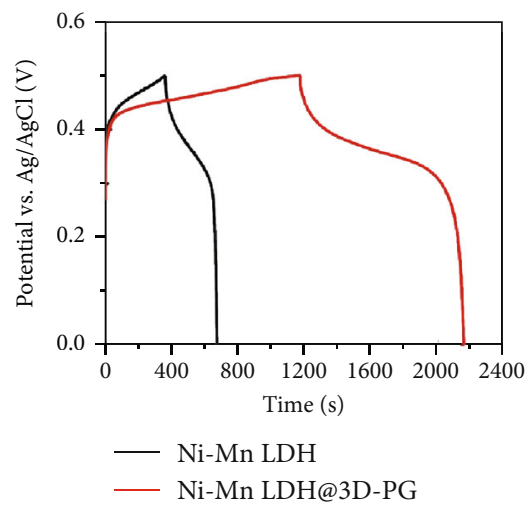

(e)

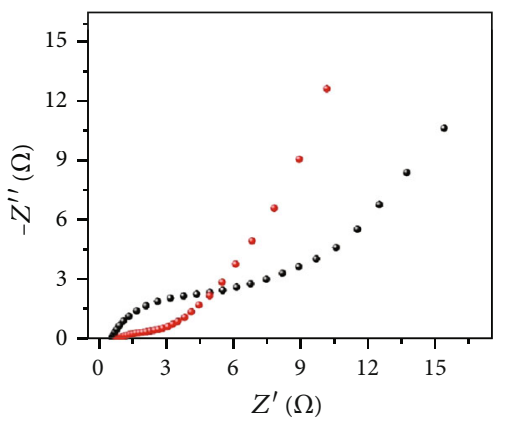

- Ni-Mn LDH

- Ni-Mn LDH@3D-PG

(h)

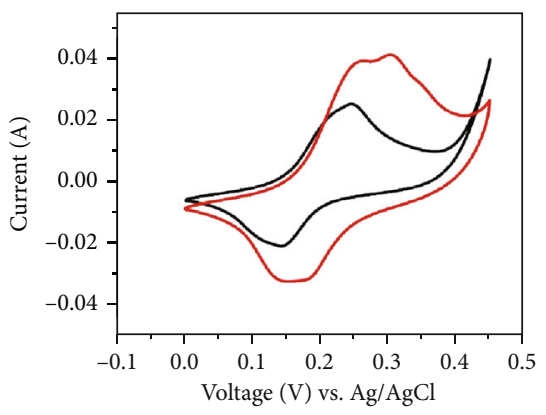

— Ni-Co LDH

—_ Ni-Co LDH@3D-PG

(c)

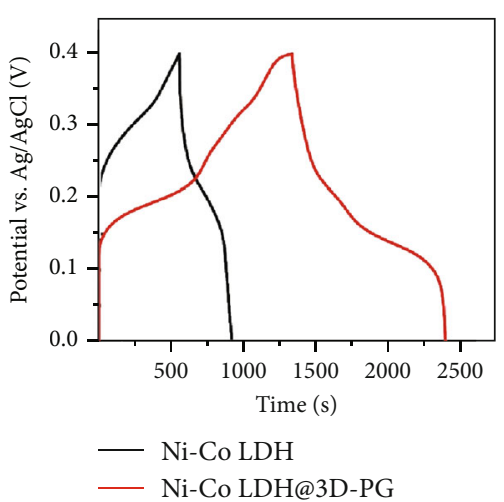

(f)

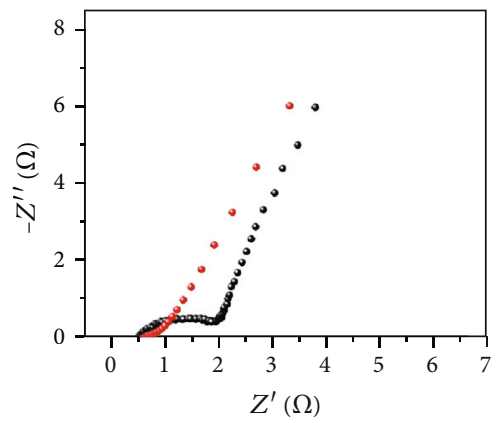

- Ni-Co LDH

- Ni-Co LDH@3D-PG

(i)

FIgure 6: Comparative cyclic voltammograms of pristine LDH and their coronal hybrids in $3 \mathrm{M}$ KOH solution using a three-electrode configuration at a scan rate of $5 \mathrm{mV} \mathrm{s}^{-1}$ for (a) Co-Mn LDH and the Co-Mn LDH@3D-PG hybrid, (b) Ni-Mn LDH and the Ni-Mn LDH@3D-PG hybrid, and (c) Ni-Co LDH and the Ni-Co LDH@3D-PG hybrid. Comparative GCD profiles for (d) Co-Mn LDH and the Co-Mn LDH@3D-PG hybrid, (e) Ni-Mn LDH and the Ni-Mn LDH@3D-PG hybrid, and (f) Ni-Co LDH and the Ni-Co LDH@3D-PG hybrid. Comparative EIS profiles obtained for (g) Co-Mn LDH and the Co-Mn LDH@3D-PG hybrid, (h) Ni-Mn LDH and the Ni-Mn LDH@3D-PG hybrid, and (i) Ni-Co LDH and the Ni-Co LDH@3D-PG hybrid.

from GCD are found to be $250 \mathrm{Cg}^{-1}, 314 \mathrm{Cg}^{-1}$, and $361 \mathrm{Fg}^{-1}$ in potentials ranging from -0.2 to $0.6 \mathrm{~V}, 0$ to $0.5 \mathrm{~V}$, and 0 to $0.4 \mathrm{~V}$, respectively, at a current density of $1 \mathrm{Ag}^{-1}$ for all the samples. The graphene-based coronal hybrids of these pristine $\mathrm{LDH}$ materials exhibited an ultrahigh specific capacity of $776 \mathrm{Cg}^{-1}, 984 \mathrm{Cg}^{-1}$, and $1056 \mathrm{Cg}^{-1}$, respectively, in the same potential window and at the same current density. Approximately, a threefold increase in specific capacity values suggest the vital role played by the morphology of nanostructures in enhancing the charge storage capacity, thereby improving the electrochemical activity of the LDHs.

To further evaluate the electrochemical performance, EIS studies were performed in an open circuit configuration with a potential of $10 \mathrm{mV}$ and a frequency range from $10 \mathrm{mHz}$ to $100 \mathrm{kHz}$ as shown in Figures $6(\mathrm{~g})-6(\mathrm{i})$. The EIS spectra of the pristine LDHs were compared with those of the coronal 


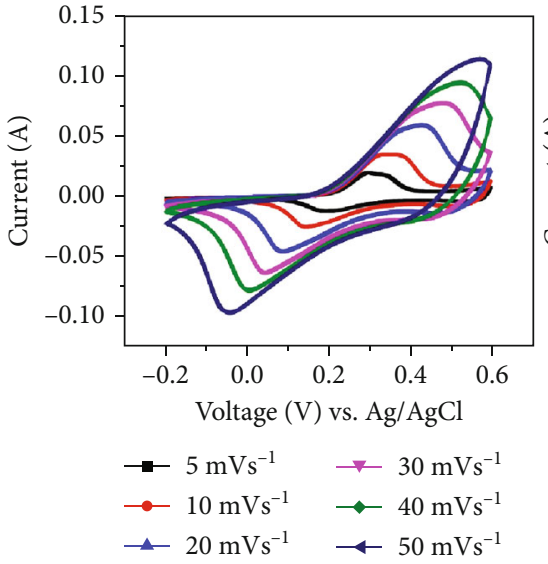

(a)
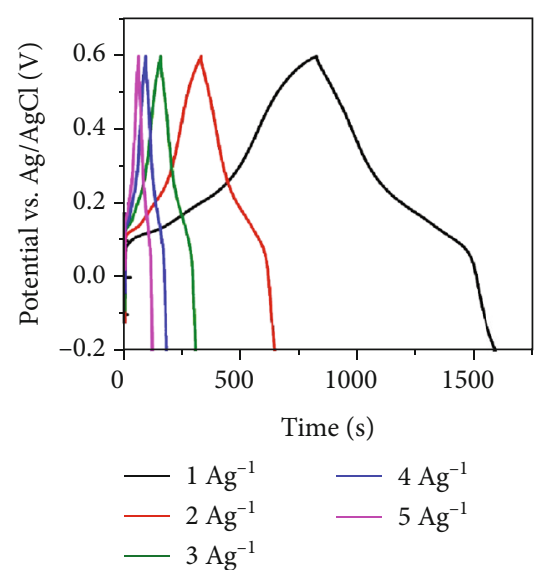

(d)

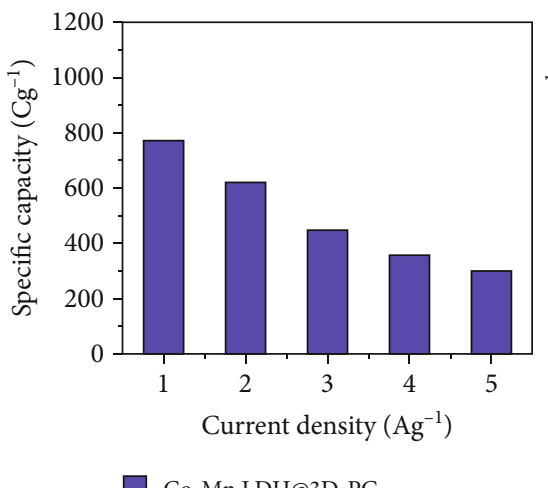

Co-Mn LDH@3D-PG

(g)

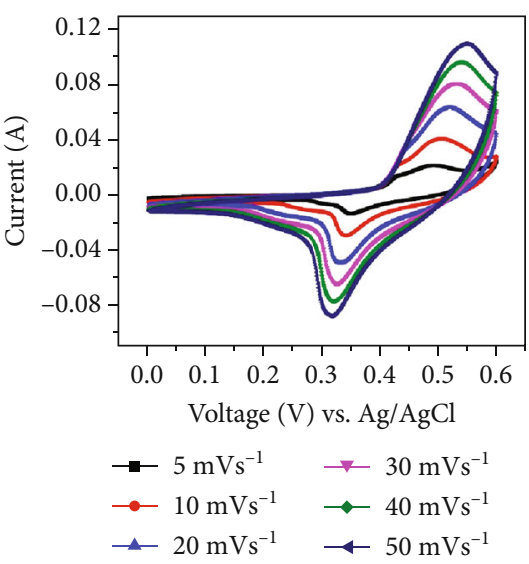

(b)
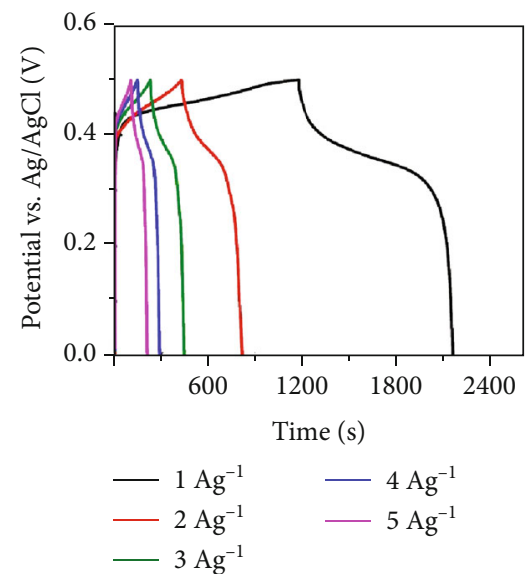

(e)

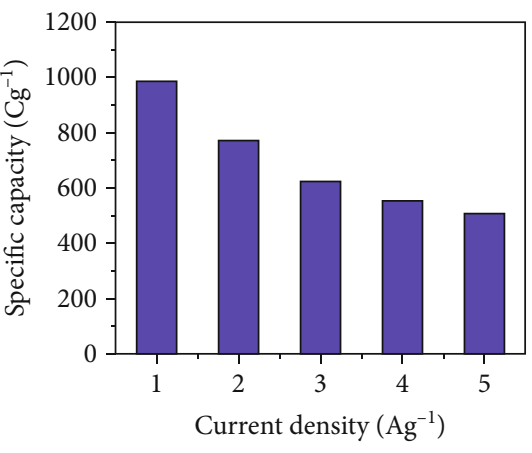

Ni-Mn LDH@3D-PG

(h)

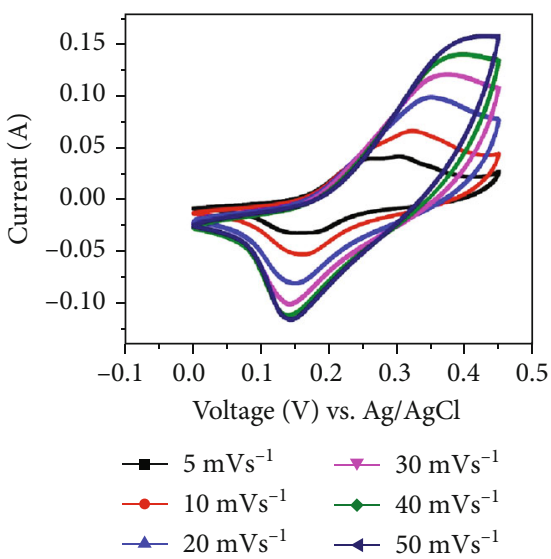

(c)
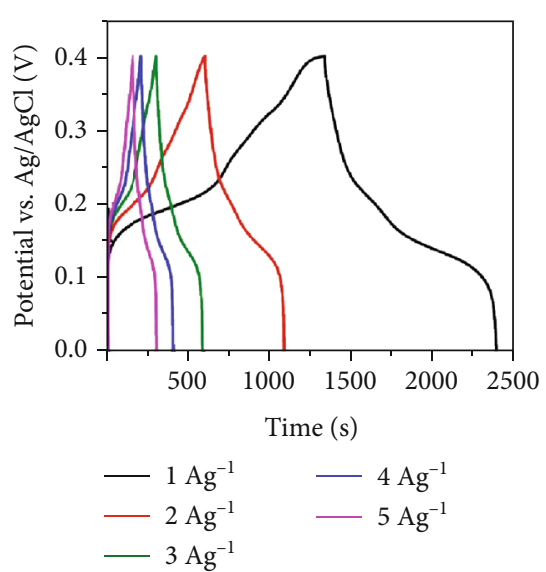

(f)

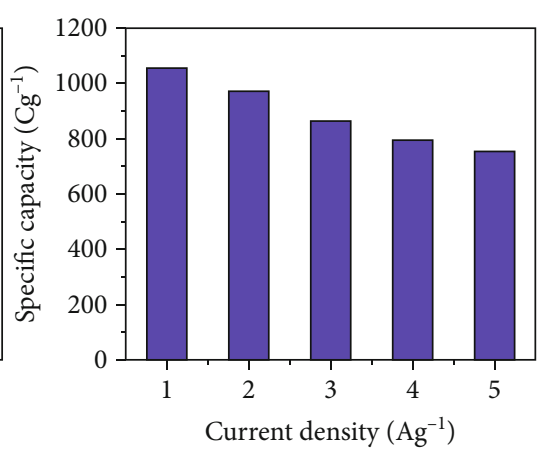

Ni-Co LDH@3D-PG

(i)

Figure 7: Cyclic voltammograms of coronal hybrids in a $3 \mathrm{M} \mathrm{KOH}$ solution using a three-electrode configuration at various scan rates for (a) Co-Mn LDH@3D-PG hybrids, (b) Ni-Mn LDH@3D-PG hybrids, and (c) Ni-Co LDH@3D-PG hybrids. GCD profiles obtained at various current densities for (d) Co-Mn LDH@3D-PG hybrids, (e) Ni-Mn LDH@3D-PG hybrids, and (f) Ni-Co LDH@3D-PG hybrids. Specific capacity vs. current density profiles obtained at various current densities for (g) Co-Mn LDH@3D-PG hybrids, (h) Ni-Mn LDH@3D-PG hybrids, and (i) Ni-Co LDH@3D-PG hybrids.

hybrids to understand the impact of morphology on the electronic and ionic transport. The Nyquist plots obtained for all the samples resembled a semicircle in the high-frequency domain specifying the charge transfer resistance $\left(R_{\mathrm{ct}}\right)$ that makes a horizontal intercept. The $R_{\mathrm{ct}}$ values for the pristine $\mathrm{LDH}$ systems are found to be approximately $6.25 \Omega, 5.05 \Omega$, and $1.45 \Omega$ for Co-Mn, Ni-Mn, and Ni-Co LDHs. It is observed that the respective coronal hybrids have $R_{\mathrm{ct}}$ values of approximately $2.70 \Omega, 2.25 \Omega$, and $0.25 \Omega$. These values show that the coronal hybrids have relatively smallerdiameter semicircles (a smaller value of $R_{\mathrm{ct}}$ ) which signify the vital role of 3D-PG networks at the electrode- 
electrolytic interface, with a low ionic resistance of electrolytic species accompanied with good electrical conductivity. Further, the EIS spectra have vertical lines with a phase inclination of above $45^{\circ}$ for both the electrode systems in the low-frequency range which demonstrate a diffusioncontrolled process, otherwise called a Warburg component. This indicates the Faradaic nature of the charge transfer process. Better ionic diffusion with shorter ionic intercalation pathways for ionic species in the alkaline electrolyte in coronal hybrids is inferred from the relatively higher inclination of the Warburg component. The overall resistance associated with active material/current collector interface and the intrinsic resistance from the electrode material in the electrolyte known from the solution resistance $\left(R_{s}\right)$ are represented as a horizontal intersection of each semicircle with the $x$-axis. The $R_{s}$ values obtained from the EIS measurements are $\sim 0.45 \Omega$, $\sim 0.55 \Omega$, and $\sim 0.59 \Omega$ for the pristine Co-Mn, Ni-Mn, and $\mathrm{Ni}-\mathrm{Co} \mathrm{LDH}$, respectively. The values of $R_{s}$ were found to be similar for the coronal hybrids as well.

Further, to give a detailed picture about the electrochemical activity of the coronal hybrids, cyclic voltammograms were recorded at various scan rates. Figures $7(a)-7$ (c) show the $\mathrm{CV}$ profiles of the three coronal hybrids obtained at scan rates of $5 \mathrm{mVs}^{-1}, 10 \mathrm{mVs}^{-1}, 20 \mathrm{mVs}^{-1}, 30 \mathrm{mVs}^{-1}, 40 \mathrm{mVs}^{-1}$, and $50 \mathrm{mVs}^{-1}$. The occurrence of redox peaks in the CV profiles describes the Faradaic nature of the electrodes. The CV profiles for the pristine materials are shown as Supplementary Figure S6. Figures 7(d)-7(f) show the GCD profiles of the coronal hybrid electrodes, obtained at various current densities. The highly reversible Faradaic charge-discharge profiles appear to be mirror images to each other at various current densities suggesting a typical battery-type performance. The coronal hybrid materials have large discharge rates compared to their pristine counterparts.

The CV and GCD profiles of coronal hybrids represent typical characteristics of a redox material with battery-type behaviour. Hence, the higher scan rates result in steeper concentration gradients, which in turn result in higher diffusive transport of the electrolytic species. The GCD profiles of pristine LDHs are shown in Supplementary Figures S6(d)S6(f) at various high current densities. An increase in the discharge current densities accompanied with a gradual decrease in the specific capacities is due to fast acting Faradaic reactions that drive incomplete utilization of electrode material. Hence, during the fast intercalation/ deintercalation process in LDHs, at higher chargedischarge rates, the $\mathrm{H}^{+}$ionic transport is limited to the solid interface, and the interaction or the reactivity of $\mathrm{H}^{+}$ with the hydroxyl ions is also limited. The coronal hybrid electrodes exhibit a maximum specific capacity at $1 \mathrm{Ag}^{-1}$ and still retains high specific capacities of $300 \mathrm{Cg}^{-1}, 505 \mathrm{Cg}^{-}$ ${ }^{1}$, and $755 \mathrm{Cg}^{-1}$ at $5 \mathrm{Ag}^{-1}$ for Co-Mn LDH@3D-PG, Ni-Mn LDH@3D-PG, and Ni-Co LDH@3D-PG, respectively, as illustrated in Figures $7(\mathrm{~g})-7(\mathrm{i})$. The cyclic stability profiles of coronal hybrids for about 4000 cycles are presented in Supplementary Figure S7. The battery-type characteristics of the graphene coronal hybrids are finely regulated with tunable interlayer spacing, composition, and interfacial chemistry of LDHs with graphene-based nanowebs. As a consequence, the enhanced battery-type property with a notable specific capacity is still persistent even at high current densities.

\section{Conclusion}

To sum up, graphene-based designer nanohybrids in the form of coronal morphologies have been synthesized with $\mathrm{Co}-\mathrm{Mn}, \mathrm{Ni}-\mathrm{Mn}$, and Ni-Co LDH lamellae self-assembled radially outwards as a corona on the surface of rGO shells connected by porous graphene networks. The well-tailored nanodesign, with a tunable interlayer spacing along with modification of the interfacial chemistry with interconnected 3D-graphene-like nanoweb structures, supplements better electronic and ionic conductivities. This in turn leads to low internal resistance that enables a fast charge transfer process with enhanced electrochemical stability. The 3DPGs are mechanically robust structures and enable the electrodes in tolerating high charging currents. This results in higher energy and power densities in coronal hybrids as compared to pristine and stacked composites reported in the literature. The efficient and strategic approach to develop coronal hybrids takes into account the tunable compositional and structural features of the transition-metallayered double hydroxide materials $(\mathrm{Ni}, \mathrm{Co}$, or $\mathrm{Mn})$ with well-connected 3D-PG nanoweb architectures that have yielded a high specific capacity. The high rate capability is understood to be due to the highly percolated 3D-PGs inside the coronal sphere with radially aligned ultrathin $\mathrm{LDH}$ nanostructures. These newly developed nanohybrids can deliver high specific capacities of $776 \mathrm{Cg}^{-1}, 984 \mathrm{Cg}^{-1}$, and $1056 \mathrm{Cg}^{-1}$ for Co-Mn LDH@3D-PG, Ni-Mn LDH@3D-PG, and Ni-Co LDH@3D-PG, respectively, with outstanding rate capability. The holistic design approach in tailoring the morphologies of energy storage materials can lead to the rational design and development of a wide range of functional materials for higher energy and power densities for energy storage applications.

\section{Data Availability}

No data was used to support this study.

\section{Conflicts of Interest}

The authors declare no conflict of financial interests or personal relationships that could give the impression of influencing this work.

\section{Acknowledgments}

We acknowledge the support of the SAIF-Indian Institute of Technology Bombay for access to instrumentation facilities.

\section{Supplementary Materials}

Figure S1: comparison of morphologies for pristine LDHs and coronal nanohybrid samples. Figure S2: comparison of high-resolution STEM micrographs for pristine LDHs and coronal nanohybrid samples. Figure S3: selected area 
diffraction patterns obtained from HR-TEM. Representation of lattice planes for (a) Co-Mn LDH@3D-PG, (b) Ni-Mn LDH@3D-PG, and (c) Ni-Co LDH@3D-PG. Figure S4: Fourier transform infrared spectra of the coronal nanohybrid samples (Co-Mn LDH@3D-PG, Ni-Mn LDH@3D-PG, and Ni-Co LDH@3D-PG) to realize the chemical structure. Figure S5: (A-C) STEM elemental maps of pristine Co-Mn $\mathrm{LDH}, \mathrm{Ni}-\mathrm{Mn} \mathrm{LDH}$, and $\mathrm{Ni}-\mathrm{CoLDH}$, respectively, with their overlay micrographs. Composite micrographs of (D) Co-Mn LDH@3D-PG, (E) Ni-Mn LDH@3D-PG, and (F) Ni-Co LDH@3D-PG. Figure S6: cyclic voltammograms of pristine LDHs in a $3 \mathrm{M} \mathrm{KOH}$ solution using a three-electrode configuration at various scan rates for (a) Co-Mn LDH, (b) Ni-Mn LDH, and (c) Ni-Co LDH. GCD profiles obtained at various current densities for (d) Co-Mn LDH, (e) Ni-Mn LDH, and (f) Ni-Co LDH. Relationship showing specific capacity vs. current density profiles obtained at various current densities for (g) Co-Mn LDH, (h) Ni-Mn LDH, and (i) Ni-Co LD. Figure S7: (a) cycle stability profile. (b, c) First and last three galvanostatic charge-discharge cycles of Co-Mn LDH@3DPG, respectively. (d) Cycle stability profile. (e, f) First and last three galvanostatic charge-discharge cycles of $\mathrm{Ni}-\mathrm{Mn}$ LDH@3D-PG, respectively. (g) Cycle stability profile. (h, i) First and last three galvanostatic charge-discharge cycles of Ni-Co LDH@3D-PG, respectively. Figure S8: electrochemical performance of $3 \mathrm{D}$ graphene-based core shell structures. (a) Cyclic voltammograms obtained at various current densities. (b) Charge-discharge profiles obtained at various current densities. (c) Specific capacities derived from cycling discharge profiles at various current densities. Table 1: comparison of coronal graphene/LDH nanohybrids with similar nanomaterials. (Supplementary Materials)

\section{References}

[1] C. Tan, X. Cao, X.-J. Wu et al., "Recent advances in ultrathin two-dimensional nanomaterials," Chemical Reviews, vol. 117, no. 9, pp. 6225-6331, 2017.

[2] K. S. Novoselov, A. Mishchenko, A. Carvalho, and A. H. Castro Neto, "2D materials and van der Waals heterostructures," Science, vol. 353, article aac9439, 2016.

[3] X. Guo, S. Zheng, G. Zhang et al., "Nanostructured graphenebased materials for flexible energy storage," Energy Storage Materials, vol. 9, pp. 150-169, 2017.

[4] S. Zhai, L. Wei, H. E. Karahan et al., "2D materials for 1D electrochemical energy storage devices," Energy Storage Materials, vol. 19, pp. 102-123, 2019.

[5] B. E. Conway, Electrochemical Supercapacitors: Scientific Fundamentals and Technological Applications, Kluwar academic/ plenum, New York, 1999.

[6] X. Cao, Z. Yin, and H. Zhang, "Three-dimensional graphene materials: preparation, structures and application in supercapacitors," Energy \& Environmental Science, vol. 7, no. 6, pp. 1850-1865, 2014.

[7] V. Rives, Layered double hydroxides: present and future, Nova Publishers, 2001.

[8] D. G. Evans and R. C. T. Slade, Structural Aspects of Layered Double Hydroxides, Springer-Verlag, Berlin/Heidelberg, 2005.

[9] P. J. Sideris, U. G. Nielsen, Z. Gan, and C. P. Grey, "Mg/Al ordering in layered double hydroxides revealed by multinu- clear NMR spectroscopy," Science, vol. 321, no. 5885, pp. 113-117, 2008.

[10] L. Zhang and M. Jaroniec, "Strategies for development of nanoporous materials with $2 \mathrm{D}$ building units," Chemical Society Reviews, vol. 49, no. 16, pp. 6039-6055, 2020.

[11] H. Cheng, Y. Huang, G. Shi, L. Jiang, and L. Qu, "Graphenebased functional architectures: sheets regulation and macrostructure construction toward actuators and power generators," Accounts of Chemical Research, vol. 50, no. 7, pp. 1663-1671, 2017.

[12] K. Khan, A. K. Tareen, M. Aslam et al., "Recent advances in two-dimensional materials and their nanocomposites in sustainable energy conversion applications," Nanoscale, vol. 11, no. 45, pp. 21622-21678, 2019.

[13] J. Yu, Q. Wang, D. O'Hare, and L. Sun, "Preparation of two dimensional layered double hydroxide nanosheets and their applications," Chemical Society Reviews, vol. 46, no. 19, pp. 5950-5974, 2017.

[14] R. Ma, Z. Liu, K. Takada, N. Iyi, Y. Bando, and T. Sasaki, "Synthesis and exfoliation of $\mathrm{Co}^{2+}-\mathrm{Fe}^{3+}$ layered double hydroxides: an innovative topochemical approach," Journal of the American Chemical Society, vol. 129, no. 16, pp. 5257-5263, 2007.

[15] P. Sun, R. Ma, X. Bai, K. Wang, H. Zhu, and T. Sasaki, “Singlelayer nanosheets with exceptionally high and anisotropic hydroxyl ion conductivity," Science Advances, vol. 3, no. 4, article e1602629, 2017.

[16] K. S. Novoselov, A. K. Geim, S. V. Morozov et al., "Twodimensional gas of massless Dirac fermions in graphene," Nature, vol. 438, no. 7065, pp. 197-200, 2005.

[17] A. K. Geim and K. S. Novoselov, "The rise of graphene," Nature Materials, vol. 6, no. 3, pp. 183-191, 2007.

[18] N. Mahmood, C. Zhang, H. Yin, and Y. Hou, "Graphene-based nanocomposites for energy storage and conversion in lithium batteries, supercapacitors and fuel cells," Journal of Materials Chemistry A, vol. 2, no. 1, pp. 15-32, 2014.

[19] F. Bonaccorso, L. Colombo, G. Yu et al., "Graphene, related two-dimensional crystals, and hybrid systems for energy conversion and storage," Science, vol. 347, no. 6217, p. 1246501, 2015.

[20] Y. Xu, Z. Lin, X. Zhong et al., "Holey graphene frameworks for highly efficient capacitive energy storage," Nature communications, vol. 5, p. 4554, 2014.

[21] S. K. Kiran, S. Shukla, A. Struck, and S. Saxena, "Surface enhanced 3D rGO hybrids and porous rGO nano-networks as high performance supercapacitor electrodes for integrated energy storage devices," Carbon, vol. 158, pp. 527-535, 2020.

[22] L. Zhi, T. Li, H. Yu et al., "Hierarchical graphene network sandwiched by a thin carbon layer for capacitive energy storage," Carbon, vol. 113, pp. 100-107, 2017.

[23] Z. Cai, X. Bu, P. Wang, J. C. Ho, J. Yang, and X. Wang, "Recent advances in layered double hydroxide electrocatalysts for the oxygen evolution reaction," Journal of Materials Chemistry $A$, vol. 7, no. 10, pp. 5069-5089, 2019.

[24] F. Song and X. Hu, "Exfoliation of layered double hydroxides for enhanced oxygen evolution catalysis," Nature Communications, vol. 5, no. 1, p. 4477, 2014.

[25] X. Chia and M. Pumera, "Characteristics and performance of two-dimensional materials for electrocatalysis," Nature Catalysis, vol. 1, no. 12, pp. 909-921, 2018. 
[26] Y. Zhai, Y. Dou, D. Zhao, P. F. Fulvio, R. T. Mayes, and S. Dai, "Carbon materials for chemical capacitive energy storage," Advanced Materials, vol. 23, no. 42, pp. 4828-4850, 2011.

[27] A. G. Pandolfo and A. F. Hollenkamp, "Carbon properties and their role in supercapacitors," Journal of Power Sources, vol. 157, no. 1, pp. 11-27, 2006.

[28] D.-W. Wang, F. Li, M. Liu, G. Q. Lu, and H.-M. Cheng, "3D aperiodic hierarchical porous graphitic carbon material for high-rate electrochemical capacitive energy storage," Angewandte Chemie International Edition, vol. 47, no. 2, pp. 373376, 2008.

[29] G. Fan, F. Li, D. G. Evans, and X. Duan, "Catalytic applications of layered double hydroxides: recent advances and perspectives," Chemical Society Reviews, vol. 43, no. 20, pp. 70407066, 2014.

[30] S. He, Z. An, M. Wei, D. G. Evans, and X. Duan, "Layered double hydroxide-based catalysts: nanostructure design and catalytic performance," Chemical Communications, vol. 49, no. 53, pp. 5912-5920, 2013.

[31] N. Baig and M. Sajid, "Applications of layered double hydroxides based electrochemical sensors for determination of environmental pollutants: a review," Trends in Environmental Analytical Chemistry, vol. 16, pp. 1-15, 2017.

[32] D. Shan, S. Cosnier, and C. Mousty, "Layered double hydroxides: an attractive material for electrochemical biosensor design," Analytical Chemistry, vol. 75, no. 15, pp. 3872-3879, 2003.

[33] V. Rives, M. del Arco, and C. Martín, "Intercalation of drugs in layered double hydroxides and their controlled release: a review," Applied Clay Science, vol. 88-89, pp. 239-269, 2014.

[34] A. C. S. Alcântara, P. Aranda, M. Darder, and E. Ruiz-Hitzky, "Bionanocomposites based on alginate-zein/layered double hydroxide materials as drug delivery systems," Journal of Materials Chemistry, vol. 20, no. 42, pp. 9495-9504, 2010.

[35] E. N. Kalali, X. Wang, and D.-Y. Wang, "Functionalized layered double hydroxide-based epoxy nanocomposites with improved flame retardancy and mechanical properties," Journal of Materials Chemistry A, vol. 3, no. 13, pp. 6819-6826, 2015.

[36] F. Leroux and J.-P. Besse, "Polymer interleaved layered double hydroxide: a new emerging class of nanocomposites," Chemistry of Materials, vol. 13, no. 10, pp. 3507-3515, 2001.

[37] G. Abellán, E. Coronado, C. Martí-Gastaldo, J. Waerenborgh, and A. Ribera, "Interplay between chemical composition and cation ordering in the magnetism of $\mathrm{Ni} / \mathrm{Fe}$ layered double hydroxides," Inorganic Chemistry, vol. 52, no. 17, pp. 1014710157, 2013.

[38] J.-H. Choy, S.-Y. Kwak, Y.-J. Jeong, and J.-S. Park, "Inorganic layered double hydroxides as nonviral vectors," Angewandte Chemie International Edition, vol. 39, no. 22, pp. 4041-4045, 2000.

[39] A. Vaccari, "Clays and catalysis: a promising future," Applied Clay Science, vol. 14, no. 4, pp. 161-198, 1999.

[40] A. I. Khan and D. O'Hare, "Intercalation chemistry of layered double hydroxides: recent developments and applications," Journal of Materials Chemistry, vol. 12, no. 11, pp. 31913198, 2002.

[41] R. Allmann, "The crystal structure of pyroaurite," Acta Crystallographica Section B: Structural Crystallography and Crystal Chemistry, vol. 24, no. 7, pp. 972-977, 1968.
[42] H. F. W. Taylor, "Segregation and cation-ordering in sjögrenite and pyroaurite," Mineralogical Magazine, vol. 37, no. 287, pp. 338-342, 1969.

[43] M. Armand and J.-M. Tarascon, "Building better batteries," Nature, vol. 451, no. 7179, pp. 652-657, 2008.

[44] A. C. Ferrari, J. C. Meyer, V. Scardaci et al., "Raman spectrum of graphene and graphene layers," Physical Review Letters, vol. 97 , no. 18, p. 187401, 2006.

[45] S. Stankovich, D. A. Dikin, R. D. Piner et al., "Synthesis of graphene-based nanosheets via chemical reduction of exfoliated graphite oxide," Carbon, vol. 45, no. 7, pp. 1558-1565, 2007.

[46] A. C. Ferrari and J. Robertson, "Interpretation of Raman spectra of disordered and amorphous carbon," Physical Review B, vol. 61, no. 20, pp. 14095-14107, 2000.

[47] S. Karthik Kiran, S. Shukla, A. Struck, and S. Saxena, "Surface engineering of graphene oxide shells using lamellar LDH nanostructures," ACS Applied Materials \& Interfaces, vol. 11, no. 22, pp. 20232-20240, 2019.

[48] Y. Zhao, F. Li, R. Zhang, D. G. Evans, and X. Duan, "Preparation of layered double-hydroxide nanomaterials with a uniform crystallite size using a new method involving separate nucleation and aging steps," Chemistry of Materials, vol. 14, no. 10, pp. 4286-4291, 2002.

[49] M. Bellotto, B. Rebours, O. Clause, J. Lynch, D. Bazin, and E. Elkaïm, "A reexamination of hydrotalcite crystal chemistry," The Journal of Physical Chemistry, vol. 100, no. 20, pp. 8527-8534, 1996.

[50] Y. Wang, Y. Song, and Y. Xia, "Electrochemical capacitors: mechanism, materials, systems, characterization and applications," Chemical Society Reviews, vol. 45, no. 21, pp. 59255950, 2016.

[51] K. K. Sarigamala, S. Shukla, A. Struck, and S. Saxena, "Rationally engineered 3D-dendritic cell-like morphologies of $\mathrm{LDH}$ nanostructures using graphene-based core-shell structures," Microsystems \& Nanoengineering, vol. 5, no. 1, p. 65, 2019.

[52] Z. Gu, J. J. Atherton, and Z. P. Xu, "Hierarchical layered double hydroxide nanocomposites: structure, synthesis and applications," Chemical Communications, vol. 51, no. 15, pp. 3024 3036, 2015.

[53] F. Zhang, T. Zhang, X. Yang et al., "A high-performance supercapacitor-battery hybrid energy storage device based on graphene-enhanced electrode materials with ultrahigh energy density," Energy \& Environmental Science, vol. 6, no. 5, pp. 1623-1632, 2013.

[54] B. Zhao, D. Chen, X. Xiong et al., "A high-energy, long cyclelife hybrid supercapacitor based on graphene composite electrodes," Energy Storage Materials, vol. 7, pp. 32-39, 2017. 